\title{
Design of a Scaled Flight Test Vehicle Including Linear Aeroelastic Effects
}

\author{
Charles A.G. Eger \\ Thesis submitted to the Faculty of the \\ Virginia Polytechnic Institute and State University \\ in partial fulfillment of the requirements for the degree of \\ Master of Science \\ in \\ Aerospace Engineering
}

Robert A. Canfield, Chair

Mayuresh J. Patil

Ned J. Lindsley

May 7, 2013

Blacksburg, Virginia

Keywords: Aeroelasticity, Scaling, Joined-wing

Copyright 2013, Charles A.G. Eger 


\title{
Design of a Scaled Flight Test Vehicle Including Linear Aeroelastic Effects
}

\author{
Charles A.G. Eger
}

\section{(ABSTRACT)}

A procedure for the design of a scaled aircraft using linear aeroelastic scaling is developed and demonstrated. Previous work has shown the viability in matching scaled structural frequencies and mode shapes in order to achieve consistent linear scaling of simple models. This methodology is adopted for use on a high fidelity joined-wing aircraft model. Natural frequencies and mode shapes are matched by optimizing structural ply properties and nonstructural mass. A full-scale SensorCraft concept developed by AFRL and Boeing serves as the target model, and a $1 / 9^{\text {th }}$ span geometrically scaled remotely piloted vehicle (RPV) serves as the initial design point. The aeroelastic response of the final design is verified against the response of the full-scale model. Reasonable agreement is seen in both aeroelastic damping and frequency for a range of flight velocities, but some discrepancy remains in accurately capturing the flutter velocity. 


\section{Acknowledgments}

I would like to thank my advisors, Dr. Robert Canfield and Dr. Mayuresh Patil for their help in producing this work. Their guidance and support has always helped lead me down the correct path, especially in the last few months of research.

I would like to thank our AFRL POC, Dr. Ned Lindsley, for his computational help in the last phase of the project. His knowledge and expertise of ZONA's ZAERO software greatly aided in performing the aeroelastic verification step.

I would also like to acknowledge Anthony Ricciardi for his constant advice and tips. He was always willing to listen to my problems and offer helpful suggestions. Anthony was always the first person I asked questions to when running into trouble.

Finally, I would like to thank my parents for their constant love and encouragement. They have always been there for me, providing support every step of the way. Nothing would have been possible without them. 


\section{Contents}

List of Figures vii

List of Tables $\quad$ x

1 Introduction $\quad 1$

2 Literature Review $\quad 4$

3 Scaling Theory and Methodology 11

3.1 Non-dimensionalizing the Equations of Motion . . . . . . . . . . . . . . 11

3.2 Calculating the Scaling Parameters . . . . . . . . . . . . . 17

4 Optimizing the Scaled Modal Response $\quad 19$

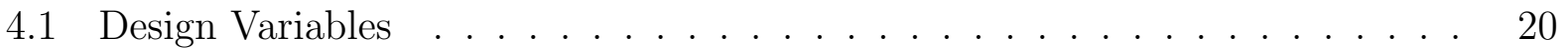

4.2 Objective Function . . . . . . . . . . . . . . . . . . 21 
4.3 Constraints . . . . . . . . . . . . . . . . . . . . . . 23

4.4 Optimization Results . . . . . . . . . . . . . . . . . . . 24

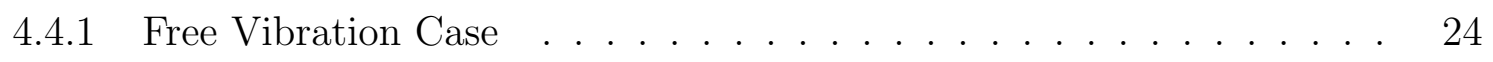

4.4 .2 Constrained Vibration Case . . . . . . . . . . . . . . 27

5 Aeroelastic Response Verification $\quad 31$

5.1 Free Vibration Aeroelastic Results . . . . . . . . . . . . . . . . . . . . 32

5.2 Constrained Vibration Aeroelastic Results . . . . . . . . . . . . . . 34

6 Conclusions and Recommendations 38

6.1 Conclusions . . . . . . . . . . . . . . . . . . . . . . 38

6.2 Recommendations . . . . . . . . . . . . . . . . . . 40

$\begin{array}{ll}\text { References } & 42\end{array}$

$\begin{array}{lr}\text { Appendices } & 46\end{array}$

$\begin{array}{lll}\text { Appendix A Finite Element Model Conversion } & 47\end{array}$

A.1 Initial ANSYS Conversion $\ldots \ldots \ldots \ldots \ldots \ldots \ldots$

A.2 Fixing the Material Properties . . . . . . . . . . . . . . 48

A.3 Fixing the Ply Element Properties . . . . . . . . . . . . . . . . . . . 49 
A.4 Fixing the Model Connections . . . . . . . . . . . . . . . . . . 51

A.5 Model Verification . . . . . . . . . . . . . . . . . 52

Appendix B High Fidelity Flight Test Modeling

B.1 Early Design Exploration . . . . . . . . . . . . . . . . . . 54

B.2 Aft Wing Ladder Structure . . . . . . . . . . . . . . . 56

B.3 Model Tuning and Forward Wing Testing . . . . . . . . . . . . . . 57 


\section{List of Figures}

1.1 Geometrically scaled RPV built to $1 / 9^{\text {th }}$ span of full-scale model. Photo provided by courtesy of the author. . . . . . . . . . . . . . . .

4.1 Node locations of the designed nonstructural masses on the scaled model. . .

4.2 Nodes of the scaled model used in the objective function to match the target mode shapes. . . . . . . . . . . . . . . . . . . . . . . . 22

4.3 First elastic mode shape comparison. . . . . . . . . . . . . . . . . . . 26

4.4 Second elastic mode shape comparison. . . . . . . . . . . . . . . . 26

4.5 Third elastic mode shape comparison. . . . . . . . . . . . . . . . 26

4.6 Fourth elastic mode shape comparison. . . . . . . . . . . . . . . . . . . . 27

4.7 Fifth elastic mode shape comparison. . . . . . . . . . . . . . . . . . 27

4.8 First constrained mode shape comparison. . . . . . . . . . . . . . . . . . . 29

4.9 Second constrained mode shape comparison. . . . . . . . . . . . . . . . . 29 
4.10 Third constrained mode shape comparison. . . . . . . . . . . . . . . . . . 29

4.11 Fourth constrained mode shape comparison. . . . . . . . . . . . . . 30

4.12 Fifth constrained mode shape comparison. . . . . . . . . . . . . 30

4.13 Sixth constrained mode shape comparison. . . . . . . . . . . . . . . 30

5.1 Aeroelastic damping from the free flutter analysis. . . . . . . . . . . 32

5.2 Aeroelastic frequency from the free flutter analysis. . . . . . . . . . . . 33

5.3 Free flutter mode shape comparison. . . . . . . . . . . . . . . . 34

5.4 Aeroelastic damping from the constrained flutter analysis. . . . . . . . 35

5.5 Aeroelastic frequency from the constrained flutter analysis. . . . . . . . . 36

5.6 Constrained flutter mode shape comparison. . . . . . . . . . . . . 37

B.1 Rotational "leaf" spring joint. . . . . . . . . . . . . . . . . . 55

B.2 2.5g trim load static deflection results with soft aft wing and spring joint. . . 55

B.3 Aft wing ladder beam structure integrated into high fidelity model. $\ldots . .57$

B.4 Wing tip deflections after model tuning. . . . . . . . . . . 58

B.5 Root deflections after model tuning. . . . . . . . . . . . . . 59

B.6 Fillet locations in forward wing. . . . . . . . . . . . . 60

B.7 Major principal strain of top ply layer at $13.8 \mathrm{~kg}$ and measurement locations. $\quad 61$ 
B.8 Leading edge strain measurements after model tuning. . . . . . . . . . . . . 62

B.9 Trailing edge strain measurements after model tuning. . . . . . . . . . . 63

B.10 Middle region strain measurements after model tuning. . . . . . . . . . . . 64 


\section{List of Tables}

4.1 Results of the free scaled model optimization. . . . . . . . . . . . . . 25

4.2 Results of the constrained scaled model optimization. . . . . . . . . . . 28

5.1 Flight densities and altitudes for aeroelastic response verification. . . . . . 31

5.2 Free flutter results. . . . . . . . . . . . . . . . . . . . . . . 34

5.3 Free flutter modal participation factors. . . . . . . . . . . . . . . 34

5.4 Constrained flutter results. . . . . . . . . . . . . . . . . 37

5.5 Constrained flutter modal participation factors. . . . . . . . . . . . 37

A.1 Results of the finite element model conversion. . . . . . . . . . . . . . 52 


\section{Chapter 1}

\section{Introduction}

The concept of aeroelastic scaling involves the design of a smaller span vehicle that has the same scaled aeroelastic response as a full-span target vehicle. The testing of these smaller scale models can be particularly beneficial in studying the flutter response of the target vehicle while keeping costs and manufacturing time low. This work aims to extend existing aeroelastic scaling methodologies in the context of an actual flight test vehicle.

The primary motivation of this work was to facilitate the development of an aeroelastic scaling technique for use on a joined-wing aircraft design. Originally proposed by Wolkovitch, ${ }^{1}$ joined-wing designs have many potential benefits over conventional designs including lighter weight and less induced drag. Further research into the subject has demonstrated that these weight optimal designs can be prone to geometric nonlinearities, particularly aft wing buckling. ${ }^{2,3}$ 
A full-scale joined-wing SensorCraft (JWSC) concept developed by AFRL and Boeing ${ }^{4}$ was geometrically scaled to produce a $1 / 9^{t h}$ span RPV by Richards et al. ${ }^{5}$ The RPV was designed to have similar rigid flight dynamics as the full-scale model and has successfully completed a flight demonstration. Figure 1.1 shows the RPV during flight testing. The SensorCraft is intended to serve as a next generation, high altitude, long endurance (HALE) platform to perform intelligence, surveillance, and reconnaissance (ISR) capabilities. The unique geometry of the joined-wings allows for the construction of advanced radar antennas into all four lifting surfaces, providing a complete, unobstructed, $360^{\circ}$ radar field. ${ }^{4}$

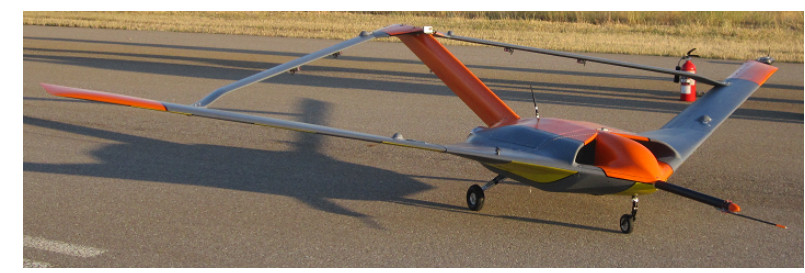

Figure 1.1: Geometrically scaled RPV built to $1 / 9^{\text {th }}$ span of full-scale model. Photo provided by courtesy of the author.

The next phase of research aims to modify the geometrically scaled design to also match the aeroelastic properties of the full-scale model. In order to fully scale the design, a methodology for matching the unique nonlinear response of the joined-wing design is required. Previous research has shown this to be an important concern in joined-wing aircraft. ${ }^{2,3}$ While matching the nonlinear response is indeed desired, this work develops a methodology for scaling the linear aeroelastic response, particularly the critical flutter condition, of the fullscale model as an initial design step. Linear scaling aided in determining the feasibility of modifying the current manufacturing method to reach an aeroelastically scaled design. 
The scaling technique used in this work is an extension of previous research. Aeroelastic scaling is achieved by matching the modal frequencies and shapes between the scaled vehicles through the optimization of a finite element model, matching the stiffness and mass distributions. A flutter analysis is used to verify the accuracy of the scaling procedure. 


\section{Chapter 2}

\section{Literature Review}

The use of scaling laws to achieve similitude between models of different size has its roots in classical dimensional analysis. In his Historico-critical Review of Dimensional Analysis, ${ }^{6}$ Macagno provides a detailed historical review of the concept. The familiar $\pi$-theorem was first formally generalized by Vaschy ${ }^{7}$ in 1892 and later again by Buckingham ${ }^{8}$ in 1914 . The theorem states that an equation with $n$ physical variables and $k$ primary dimensions can be rewritten using $p=n-k$ non-dimensional terms. By matching these non-dimensional terms between scaled models, physical similitude is produced. The process of non-dimensionalizing governing equations is the basis of all scaling theory.

The concept of applying scaling theory to aeroelastic applications is outlined by Bisplinghoff et al. in Aeroelasticity. ${ }^{9}$ Examples of how to non-dimensionalize the aeroelastic equations are given. In a typical mechanics problem, the primary dimensions are mass, 
length, and time, though other combinations of these are also common. The number of primary dimensions is equal to the number of scale factors that must be chosen to relate the models. The main design challenge of aeroelastic scaling is matching the stiffness and mass distributions between the models. It is noted that a scaled flutter model is usually not suited to also act as a structural model, since the mass requirements in each case are different.

Further insight into aeroelastic scaling is given by Molyneux in Aeroelastic Modelling. ${ }^{10}$ Using classical scaling theory, the equations of an aerothermoelastic system are non-dimensionalized. In order to achieve perfect aerothermoelastic similarity, the full and scaled models would have to be built exactly the same. By relaxing some of the non-dimensional requirements, a more practical scaling theory can be produced. For example, if the flow regime of the models is considered to be incompressible, Mach number matching can be neglected. This is a safe assumption when the maximum velocity of the flow is small relative to the local speed of sound. The Reynolds number is related to the boundary layer and heat transfer along the vehicle surface and is generally of little importance to aeroelastic effects. Neglecting to match the Reynolds number makes flow density a free parameter in the scaling problem. Using an internal beam structure to achieve the required stiffness properties is proposed as one possible design solution.

Another extensive look into aeroelastic modeling is given by Wolowicz et al. in their NASA Technical Paper Similitude Requirements and Scaling Relationships as Applied to Model Testing. ${ }^{11}$ Using classical theory, scaling requirements for different model tests are outlined. The section on flutter scaling states the requirement to correctly scale mass, inertia, 
and stiffness between the models. Considerations for actually testing built models inside of a wind tunnel are also made. This work serves as a good reference for what the scaling requirements for a given problem are, but little is offered on how to actually achieve these requirements within a model.

Modern work has extended the classic aeroelastic scaling theory to a range of different applications. Friedmann et al. outlined a method for aeroservoelastic scaling in which control laws enter into the governing equations. ${ }^{12}$ Control laws and servos often make the aeroelastic system nonlinear, requiring a more rigorous approach to ensure accurate scaling. A computer simulation model was used to account for the added requirements. The technique was successfully applied to a baseline model to produce an aeroelastically scaled model. The scaling was verified using root locus plots, flutter response, and divergence response.

Pototzky has also extended the classic aeroelastic scaling theory to include servoelastic effects. ${ }^{13}$ He provides a modal approach to scaling the equations of motion, avoiding applying a complicated scaling routine to a detailed finite element model. Applying scale factors to the finite element model as a whole would be difficult and computationally intensive. Scaling rules were instead applied to the modal matrices of the model. Scaling of the generalized modal mass matrix was assumed to provide correct scaling of the normal mode shapes. The procedure was verified by comparing root locus plots and time histories of control simulations between a full-scale model and a wind tunnel model. This method to aeroelastic scaling is of particular interest to the work of this thesis which also uses a modal approach to nondimensionalize the equations of motion. 
The concept of achieving aeroelastic scaling through the optimization of a finite element model has been in development for years. An early example of this work is provided by French et al. ${ }^{14}$ A finite element model was scaled by matching the stiffness and mass distributions in separate optimization routines. Stiffness was first matched by reducing the error between target and current flexibility matrix elements at chosen grid points by changing the dimensions of structural elements within the model. Mass distribution was then matched by optimizing the converged stiffness model by changing non-structural concentrated masses. The error in the modal deflections was minimized while constrained to match the desired modal frequencies. Attempting to only match the modal frequencies produced poorly matching mode shapes. The technique was verified on an example problem, producing a model with a predicted flutter response that matched the full-scale response very well. This work demonstrated the feasibility of designing physical properties and dimensions of a finite element model to match a desired response rather than modifying the system matrices directly.

Richards et al. ${ }^{15}$ extended the work of French et al. ${ }^{14}$ and proposed applying the uncoupled optimization technique to a scaled joined-wing SensorCraft design. Instead of matching flexibility matrix elements in the stiffness optimization step, static deflections from the target model were matched. Matching the scaled static deflection response was assumed to correctly match the stiffness distribution. The modal response was then matched by designing concentrated masses in a second optimization routine. The technique was successfully applied to a simplified test case using a cantilevered wing design. Again, the feasibility of op- 
timizing a finite element model to produce an aeroelastically scaled design was demonstrated by this work.

Pereira et al. ${ }^{16}$ provided a different approach to matching the stiffness and mass distributions of a scaled model. Instead of performing the optimization in two steps, the stiffness and mass distributions were matched in the same optimization routine by matching the modal response. The scaled natural frequencies of a joined-wing model were optimized to match a full-scale design. Mode shapes did not enter the design process. The design variables of the problem were the all of the rib thicknesses of the model. This technique resulted in a normalized flutter speed that matched the full-scale design, but the aeroelastic frequencies did not match around or above the flutter speed. Manufacturability was a concern during the design process, because the scaled design was actually built and ground tested. The modal response of the built wind tunnel model matched well the expected scaled results. This work demonstrated the feasibility of using finite element optimization to design and build a scaled wind tunnel model.

Bond et al. ${ }^{17}$ extended the work of Pereira et al. ${ }^{16}$ and attempted to add a matching nonlinear geometric stiffness to the scaling problem. Mass and stiffness distributions were again matched in the same optimization step, but mode shapes were now included in the problem formulation. Buckling eigenvalues and mode shapes were also included in an attempt to capture the nonlinear response. A modified Goland wing with an attached strut was used to verify the technique. The first three natural frequencies and mode shapes and critical buckling eigenvalue were matched between the scaled and target model. The resulting 
aeroelastic frequency and damping matched well throughout the velocity profile, but the nonlinear static response only matched up to $60 \%$ of the buckling load. In addition, it was demonstrated that matching mode shapes was required for a matching aeroelastic response.

Work has continued in an attempt to better capture the nonlinear response of the target model while keeping a matching aeroelastic response. Ricciardi et al. ${ }^{18}$ extended the work of Bond et al. ${ }^{17}$ by employing a more rigorous approach to matching this nonlinear response. Using a beam model representative of the joined-wing SensorCraft design, nonlinear static deflections were matched for various loading conditions to account for geometric nonlinearities. A second optimization was performed to match the modal response by designing the non-structural mass. The aeroelastic frequency and damping and nonlinear static response of the models were matched successfully.

A different technique for performing nonlinear aeroelastic scaling was provided by Wan et al. ${ }^{19}$ Rather than using optimization to design the structural components of the model, the required non-dimensional matrices of the system were scaled directly by matching scaled cross-sectional properties. The technique was successfully employed on a model of a very flexible aircraft. The importance of matching the Froude number in aeroelastic scaling problems was also demonstrated. This work showed that with complete access to the system matrices of the problem, nonlinear aeroelastic scaling by this analytical method is effective and accurate. The complete internal structure of the target model is needed to employ this technique, data not publicly available for the joined-wing SensorCraft model.

The work presented in this thesis is mainly an extension of the work by Bond et al. ${ }^{17}$ 
The primary goal was to extend the direct modal matching technique to a built-up, free-free flight test vehicle. For the linear scaling procedure presented here, buckling eigenvalues and mode shapes were not included in the formulation. 


\section{Chapter 3}

\section{Scaling Theory and Methodology}

\subsection{Non-dimensionalizing the Equations of Motion}

The first step to any scaling problem is to non-dimensionalize the governing equations of motion. For this work, a modal approach to scaling is developed based on the transient linear aeroelastic equations of motion

$$
[\mathbf{M}]\{\ddot{\mathbf{x}}\}+[\mathbf{K}]\{\mathrm{x}\}=\left[\mathbf{A}_{\mathbf{k}}\right]\{\mathrm{x}\}+\left[\mathbf{A}_{\mathbf{c}}\right]\{\dot{\mathrm{x}}\}+\left[\mathbf{A}_{\mathbf{m}}\right]\{\ddot{\mathbf{x}}\}+[\mathbf{M}]\left\{\mathrm{a}_{\mathrm{g}}\right\}
$$

where $\{\mathrm{x}\}$ is a vector of elastic and rigid body degrees of freedom, $[\mathbf{M}]$ is the mass matrix, $[\mathbf{K}]$ is the stiffness matrix, $\left[\mathbf{A}_{\mathbf{k}}\right],\left[\mathbf{A}_{\mathbf{c}}\right]$, and $\left[\mathbf{A}_{\mathbf{m}}\right]$ are the aerodynamic matrices, and $\left\{\mathrm{a}_{\mathrm{g}}\right\}$ is a vector containing gravitational accelerations for each degree of freedom. To non-dimensionalize the system, the components of each coefficient matrix must have unified dimensions. Coeffi- 
cient matrix dimensions are unified using a dimensional transformation matrix, $[\mathbf{T}]$, that is derived to non-dimensionalize the physical coordinate vector. For example, if the physical coordinates are two displacement and two rotation degrees of freedom

$$
\{\mathrm{x}\}=\left\{\begin{array}{l}
h_{1} \\
\theta_{1} \\
h_{2} \\
\theta_{2}
\end{array}\right\}
$$

then a non-dimensional vector of physical coordinates, $\{\overline{\mathrm{x}}\}$, is attained using

$$
\{\mathrm{x}\}=[\mathbf{T}]\{\overline{\mathrm{x}}\}=\left[\begin{array}{cccc}
b & 0 & 0 & 0 \\
0 & 1 & 0 & 0 \\
0 & 0 & b & 0 \\
0 & 0 & 0 & 1
\end{array}\right]\left\{\begin{array}{c}
h_{1} / b \\
\theta_{1} \\
h_{2} / b \\
\theta_{2}
\end{array}\right\}=\left\{\begin{array}{c}
h_{1} \\
\theta_{1} \\
h_{2} \\
\theta_{2}
\end{array}\right\}
$$

The coefficient matrices are uniformly dimensionalized by pre-multiplying by $[\mathbf{T}]^{T}$ and postmultiplying by $[\mathbf{T}]$. Take the mass matrix for example

$$
[\breve{\mathbf{M}}]=[\mathbf{T}]^{T}[\mathbf{M}][\mathbf{T}]
$$

where $\breve{()}$ denotes a matrix with uniform dimensions. By substituting for $\{\mathrm{x}\}$ with the general form of Eq. 3.3 and pre-multiplying by $[\mathbf{T}]^{T}$ the equations of motion are uniformly 
dimensionalized.

$$
[\breve{\mathbf{M}}]\{\ddot{\ddot{x}}\}+[\breve{\mathbf{K}}]\{\overline{\mathbf{x}}\}=\left[\breve{\mathbf{A}}_{\mathbf{k}}\right]\{\overline{\mathbf{x}}\}+\left[\breve{\mathbf{A}}_{\mathbf{c}}\right]\{\dot{\overline{\mathbf{x}}}\}+\left[\breve{\mathbf{A}}_{\mathbf{m}}\right]\{\ddot{\ddot{\mathbf{x}}}\}+[\breve{\mathbf{M}}]\left\{\breve{a}_{\mathrm{g}}\right\}
$$

Each term in Eq. 3.5 is has units of force $\times$ length. Non-dimensional deformations in terms of the modal coordinates are

$$
\{\overline{\mathrm{x}}\}=[\Phi]\{\eta\}
$$

where $\{\eta\}$ is the vector of model coordinates and $[\Phi]$ is the matrix of non-dimensional mode shapes. The first six mode shapes are rigid body deformations in a free vibration case. Mass and stiffness matrices are diagonalized using the bi-orthogonality property

$$
\begin{aligned}
\langle\mathbf{m}\rangle & =[\Phi]^{T}[\breve{\mathbf{M}}][\Phi] \\
\langle\mathbf{k}\rangle & =[\Phi]^{T}[\breve{\mathbf{K}}][\Phi]=\left\langle\mathbf{m} \omega^{2}\right\rangle
\end{aligned}
$$

where $\langle\omega\rangle$ is the diagonal matrix of modal frequencies. Aerodynamic coefficient matrices are modified equivalently

$$
\begin{array}{r}
{\left[\mathbf{a}_{\mathbf{k}}\right]=[\Phi]^{T}\left[\breve{\mathbf{A}}_{\mathbf{k}}\right][\Phi]} \\
{\left[\mathbf{a}_{\mathbf{c}}\right]=[\Phi]^{T}\left[\breve{\mathbf{A}}_{\mathbf{c}}\right][\Phi]} \\
{\left[\mathbf{a}_{\mathbf{m}}\right]=[\Phi]^{T}\left[\breve{\mathbf{A}}_{\mathbf{m}}\right][\Phi]}
\end{array}
$$


Using modal coordinates, the equations of motion are

$$
\begin{aligned}
\langle\mathbf{m}\rangle\{\ddot{\eta}\} & +\left\langle\mathbf{m} \omega^{2}\right\rangle\{\eta\}= \\
& {\left[\mathbf{a}_{\mathbf{k}}\right]\{\eta\}+\left[\mathbf{a}_{\mathbf{c}}\right]\{\dot{\eta}\}+\left[\mathbf{a}_{\mathbf{m}}\right]\{\ddot{\eta}\} } \\
+ & \langle\mathbf{m}\rangle[\Phi]^{-1}\left\{\breve{a}_{\mathrm{g}}\right\}
\end{aligned}
$$

Dimensions of the aerodynamic matrices and acceleration vector are moved outside as scalar coefficients

$$
\begin{aligned}
\langle\mathbf{m}\rangle\{\ddot{\eta}\}+ & \left\langle\mathbf{m} \omega^{2}\right\rangle\{\eta\}= \\
& \frac{1}{2} \rho V^{2} S b\left(\left[\overline{\mathbf{a}}_{\mathbf{k}}\right]\{\eta\}+\frac{b}{V}\left[\overline{\mathbf{a}}_{\mathbf{c}}\right]\{\dot{\eta}\}+\frac{b^{2}}{V^{2}}\left[\overline{\mathbf{a}}_{\mathbf{m}}\right]\{\ddot{\eta}\}\right) \\
+ & \frac{g}{b}\langle\mathbf{m}\rangle[\Phi]^{-1}\left\{\overline{\mathbf{a}}_{\mathrm{g}}\right\}
\end{aligned}
$$

where barred aerodynamic matrices are non-dimensional. Mass is non-dimensionalized using $\mathrm{m}_{1}$, modal mass (uniformly dimensionalized as inertia) of the first mode.

$$
\begin{aligned}
\langle\overline{\mathbf{m}}\rangle\{\ddot{\eta}\}+ & \left\langle\overline{\mathbf{m}} \boldsymbol{\omega}^{2}\right\rangle\{\eta\}= \\
& \frac{1}{2} \frac{\rho S b}{\mathrm{~m}_{1}} V^{2}\left(\left[\overline{\mathbf{a}}_{\mathbf{k}}\right]\{\eta\}+\frac{b}{V}\left[\overline{\mathbf{a}}_{\mathbf{c}}\right]\{\dot{\eta}\}+\frac{b^{2}}{V^{2}}\left[\overline{\mathbf{a}}_{\mathbf{m}}\right]\{\ddot{\eta}\}\right) \\
+ & \frac{g}{b}\langle\overline{\mathbf{m}}\rangle[\Phi]^{-1}\left\{\overline{\mathbf{a}}_{\mathrm{g}}\right\}
\end{aligned}
$$


where $\langle\overline{\mathbf{m}}\rangle$ is a diagonal matrix of non-dimensional masses with $\overline{\mathrm{m}}_{1}=1$. Time is nondimensionalized using $\omega_{1}$, the modal frequency of the first mode

$$
\begin{aligned}
\langle\overline{\mathbf{m}}\rangle\left\{\hat{\eta}^{\star}\right\} & +\left\langle\overline{\mathbf{m}} \overline{\boldsymbol{\omega}}^{\mathbf{2}}\right\rangle\{\eta\}= \\
& \frac{1}{2} \frac{\rho S b}{\mathrm{~m}_{1}} \frac{V^{2}}{\omega_{1}^{2}}\left(\left[\overline{\mathbf{a}}_{\mathbf{k}}\right]\{\eta\}+\frac{\omega_{1} b}{V}\left[\overline{\mathbf{a}}_{\mathbf{c}}\right]\{\hbar\}+\frac{\omega_{1}^{2} b^{2}}{V^{2}}\left[\overline{\mathbf{a}}_{\mathbf{m}}\right]\left\{{ }^{\star} \eta\right\}\right) \\
+ & \frac{g}{\omega_{1}^{2} b}\langle\overline{\mathbf{m}}\rangle[\Phi]^{-1}\left\{\overline{\mathbf{a}}_{\mathrm{g}}\right\}
\end{aligned}
$$

where $\langle\bar{\omega}\rangle$ is a diagonal matrix of non-dimensional modal frequecies, and $\stackrel{\star}{(})^{*}$ denotes the derivative with respect to non-dimensional time $\tau=t \omega_{1}$. Multiplying the aerodynamic term by $b^{2} / b^{2}$ and the gravitational term by $b V^{2} / b V^{2}$

$$
\begin{aligned}
& \langle\overline{\mathbf{m}}\rangle\left\{{ }^{\star *}\right\}+\left\langle\overline{\mathbf{m}} \overline{\boldsymbol{\omega}}^{2}\right\rangle\{\eta\}=
\end{aligned}
$$

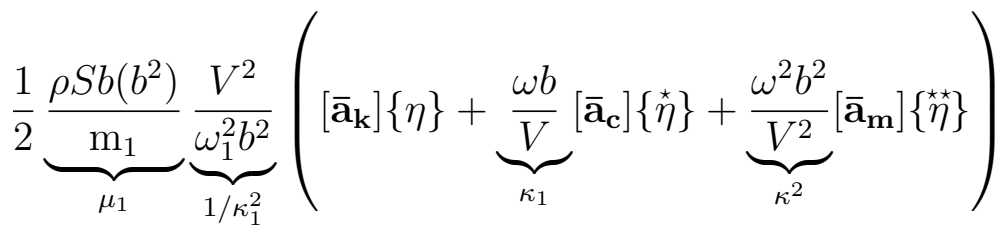

$$
\begin{aligned}
& +\underbrace{\frac{g b}{V^{2}}}_{1 / F r^{2}} \underbrace{\frac{V^{2}}{\omega_{1}^{2} b^{2}}}_{1 / \kappa_{1}^{2}}\langle\overline{\mathbf{m}}\rangle[\Phi]^{-1}\left\{\overline{\mathrm{a}}_{\mathrm{g}}\right\}
\end{aligned}
$$

three non-dimensional parameters emerge: reduced frequency $\kappa_{1}=\frac{\omega_{1} b}{V}$, an inertia ratio $\mu_{1}=\frac{\rho S b\left(b^{2}\right)}{\mathrm{m}_{1}}$, and Froude number $F r=\frac{V}{\sqrt{b g}}$. The final form of the non-dimensional aeroelastic 
equations is

$$
\begin{aligned}
\langle\overline{\mathbf{m}}\rangle\{\stackrel{\star \star}{\eta}\} & +\left\langle\overline{\mathbf{m}} \overline{\boldsymbol{\omega}}^{2}\right\rangle\{\eta\}= \\
& \frac{1}{2} \frac{\mu_{1}}{\kappa_{1}^{2}}\left(\left[\overline{\mathbf{a}}_{\mathbf{k}}\right]\{\eta\}+\kappa_{1}\left[\overline{\mathbf{a}}_{\mathbf{c}}\right]\left\{{ }_{\eta}^{\star}\right\}+\kappa_{1}^{2}\left[\overline{\mathbf{a}}_{\mathbf{m}}\right]\left\{{ }_{\eta}^{\star *}\right\}\right) \\
& +\frac{1}{F r^{2} \kappa_{1}^{2}}\langle\overline{\mathbf{m}}\rangle[\Phi]^{-1}\left\{\overline{\mathbf{a}}_{\mathrm{g}}\right\}
\end{aligned}
$$

For the scaled model to have a matching linear aeroelastic and flight dynamic response, the following are required to match the full-scale model: ${ }^{18}$

1. Reduced frequency of the first mode $\kappa_{1}$

2. Inertia ratio $\mu_{1}$

3. Froude number $\mathrm{Fr}$

4. Non-dimensional modal masses $\langle\overline{\mathbf{m}}\rangle$

5. Non-dimensional modal frequencies $\langle\bar{\omega}\rangle$

6. Non-dimensional mode shapes $[\Phi]$

7. Aerodynamic shape

8. Mach number if compressibility effects are important

9. Reynolds number if viscous effects are important 


\subsection{Calculating the Scaling Parameters}

Items 1, and 4-6 in the enumerated list from the previous section are matched through an optimization of the modal response of the scaled model, detailed in the next chapter. Items 2 and 3 are matched through the correct choice of scaling parameters. Items 7-9 are required for matching the aerodynamic matrices. Scaling of compressibility and viscous effects can be neglected with the assumption they will have a small influence for the present problem. Thus, the Mach number and Reynolds number will not be matched within this scaling procedure.

The length ratio is specified as $1 / 9^{t h}$ for the scaled flight test model. The aerodynamic shape of the target model, Item 7 , is scaled directly using this ratio. The subscript $w$ indicates the joined-wing target model, and the subscript $m$ indicates the scaled model.

$$
\lambda=\frac{b_{m}}{b_{w}}=\frac{1}{9}
$$

The airspeed ratio between the target and scaled models is selected to match the Froude number, Item 3.

$$
\begin{gathered}
F r=\frac{V_{m}}{\sqrt{b_{m} g}}=\frac{V_{w}}{\sqrt{b_{w} g}} \\
\frac{V_{m}}{V_{w}}=\sqrt{\frac{b_{m}}{b_{w}}}=\sqrt{\lambda}=\frac{1}{3}
\end{gathered}
$$

The combination of length ratio and velocity ratio determine the time scale, setting the 
frequency ratio.

$$
\frac{\omega_{m}}{\omega_{w}}=\frac{T_{w}}{T_{m}}=\frac{b_{w} / V_{w}}{b_{m} / V_{m}}=\frac{1}{\sqrt{\lambda}}=3
$$

The total mass $M$ and air density of the models must provide a matching inertia ratio, Item 2. Choosing either the mass ratio or the density ratio sets the other parameter.

$$
\begin{gathered}
\mu=\frac{\rho_{m} S_{m} b_{m}^{3}}{m_{m}}=\frac{\rho_{w} S_{w} b_{w}^{3}}{m_{w}} \\
\frac{\rho_{m} b_{m}^{3}}{M_{m}}=\frac{\rho_{w} b_{w}^{3}}{M_{w}} \\
\frac{\rho_{m}}{\rho_{w}}=\left(\frac{b_{w}}{b_{m}}\right)^{3} \frac{M_{m}}{M_{w}}=\left(\frac{1}{\lambda}\right)^{3} \frac{M_{m}}{M_{w}}=729 \frac{M_{m}}{M_{w}}
\end{gathered}
$$

Using these scaling equations $(3.18-3.24)$, any parameter from the target or scaled model can be transformed into the other scale regime. By matching the modal frequencies and shapes through an optimization problem according to these scaling equations, a linearly scaled model with aeroelastic effects is produced. 


\section{Chapter 4}

\section{Optimizing the Scaled Modal}

\section{Response}

A finite element model of the full-scale Boeing SensorCraft concept provided by AFRL ${ }^{4}$ serves as the target of the scaling procedure, and a high fidelity finite element model of the geometrically scaled RPV provided by Richards et al. serves as the initial design point. ${ }^{5}$ The initial finite element model provided was coded in ANSYS. Appendix A details the conversion process performed to create an acceptably equivalent model in Nastran.

The scaled RPV model was built to be aeroelastically stiff when compared to the fullscale model. In order to capture the scaled linear aeroelastic response, the modal response of the scaled model was matched to the Boeing target model through an optimization routine. Nastran finite element software was used to perform the optimization process. The following 
sections detail the formulation of the optimization problem.

Two separate modal optimizations were performed during the scaling procedure. The first was a free vibration case representative of the vehicle in flight. The second was a constrained vibration case more representative of a stationary wind tunnel test. The constrained vibration case was originally introduced after the free case had trouble matching the target center of gravity location and pitch inertia. While some of these matching problems have been alleviated, the scaling procedure was still carried out using both of the boundary conditions.

\subsection{Design Variables}

The skins of the geometrically scaled RPV consist mainly of carbon fiber ply layups. The internal structure consists of carbon fiber surrounding a foam core. ${ }^{5}$ The initial design variables of the optimization were the wings, tail boom, and substructure composite ply layer thicknesses and nonstructural ballast masses placed throughout the model. Figure 4.1 shows the locations of the concentrated nonstructural masses that were designed. 


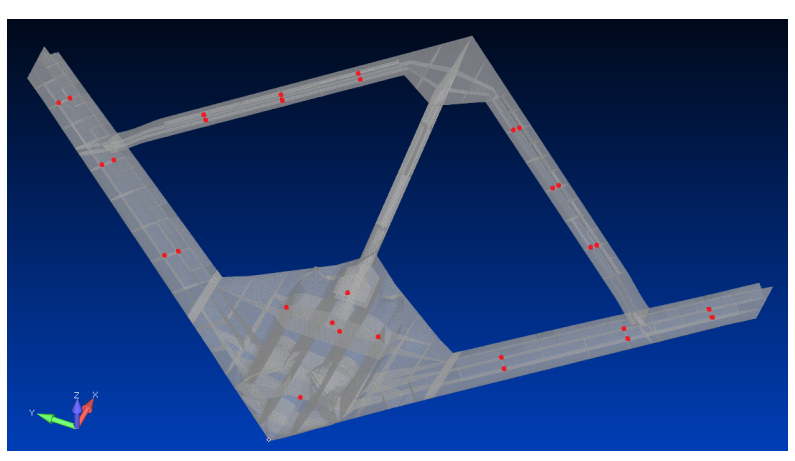

Figure 4.1: Node locations of the designed nonstructural masses on the scaled model.

These initial design variables did not provide acceptable mode shapes. Ply layer thickness design variables in the fuselage of the model were added in an attempt to better capture the modal response. In addition, movable concentrated nonstructural masses were added to the fuselage and tail boom strake. The location and value of these masses were added as design variables.

Loose bounds were placed on all of the design variables to give the optimization as much design space as possible. The minimum skin thickness for a given ply layer was set to 0.001 $m m$, and no bounds were placed on the values of the concentrated masses.

\subsection{Objective Function}

An aeroelastic analysis at sea level density of the joined-wing structure in free flight including the first thirty elastic modes indicated that the critical flutter mechanism was mainly comprised of the fifth mode. This same analysis using only the first five elastic modes reduced the flutter velocity by $6.3 \%$. Thus, it was assumed that matching the first five elastic 
modes would sufficiently capture the critical flutter mechanism. Normalized mode shapes of the first five elastic modes were extracted from the modal response of the target model and matched in the optimization routine. The modal deflections from a selected set of publicly cleared nodes shown in Figure 4.2 formed the objective function, Equation 4.1. A similar aeroelastic analysis was used to determine that the first six elastic modes should be matched in the constrained flutter case. There was a $16.1 \%$ difference in the flutter velocity between using thirty and six modes in the analysis. Thus, an additional mode was added to the objective function in the constrained case.

$$
\min \left(\sum_{i=1}^{5 \text { or } 6} W_{i}\left\|\{\bar{\phi}\}_{w, i}-\{\bar{\phi}\}_{m, i}\right\|\right)
$$

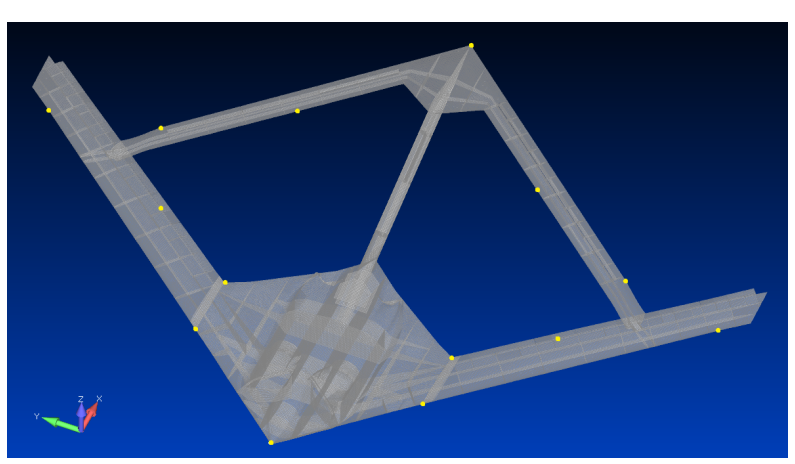

Figure 4.2: Nodes of the scaled model used in the objective function to match the target mode shapes.

Here, $\{\bar{\phi}\}_{w, i}$ is a subset of the $i^{t h}$ normalized mode shape of the full-scale target model, $\{\bar{\phi}\}_{m, i}$ is a subset of the $i^{\text {th }}$ normalized mode shape of the scaled RPV model, and $W_{i}$ is the weighting factor for the $i^{\text {th }}$ mode. The subset of the normalized mode shapes used 
correspond to the matched nodes shown in Figure 4.2. Modal deflections in the $\mathrm{x}, \mathrm{y}$, and $\mathrm{z}$ translational directions from this subset of nodes were used in this objective function. No rotational degrees of freedom were used in the objective function. It was assumed that wing twist could be captured by matching the translational degrees of freedom of matched nodes along both the leading and trailing edges of the structure.

The aeroelastic analysis of the free vibration case indicated that the critical flutter mechanism was mainly comprised of the fourth and fifth elastic modes. Thus, the fourth and fifth mode shapes were weighted more heavily than the rest of the shapes at $W_{4}=9.0$ and $W_{5}=10.0$. The first elastic mode shape was weighted at $W_{1}=7.0$ due to large initial error in the first mode shape. The remaining two mode shapes were weighted at $W_{2,3}=1.0$. The constrained vibration case was originally introduced in an effort to alleviate significant differences in pitch rotation seen in the free case. Thus, all of the mode shapes in the constrained case were weighted equally at $W_{i}=1.0$ in order to form a baseline for comparison. Changing these weights in both cases would cause certain mode shapes to match better than others.

\subsection{Constraints}

The optimization was constrained such that the normal frequencies of the target and scaled models obeyed the ratio in Equation 3.21 to within $\pm 2 \%$. No constraint was placed on the total mass of the scaled vehicle. Thus, the density ratio was set by the resulting optimized mass using Equation 3.24 . 
Constraints were later added to match the scaled center of gravity location and pitch inertia to within $\pm 5 \%$ of the target model in an attempt to better match the mode shapes in the free vibration case. Without these constraints, extraneous rigid body pitch rotation was present in some of the mode shapes. It was originally assumed that matching the modal frequencies and shapes would correctly place the center of gravity and inertias within the optimization routine, but this was not the case. These mass property constraints were not required in the constrained vibration case since the extraneous rigid body pitch rotation was removed by nature of the boundary conditions. Better matching of the mode shapes was achieved without these constraints. While the global rigid body pitch problem was alleviated with this constrained boundary condition, local pitch effects in regions such as the tail boom strake were still a concern.

\subsection{Optimization Results}

\subsubsection{Free Vibration Case}

Table 4.1 shows the results of the free vibration optimization routine. With no constraints on the total mass, the first five elastic frequencies were successfully matched to within $\pm 4.6 \%$. The third and fifth elastic frequencies were not able to be matched as closely as desired. The center of gravity location and pitch inertia were successfully matched to within $\pm 4.8 \%$. Equation 3.24 was used to set the density ratio using the unconstrained total mass of the 
scaled model, approximately $413 \mathrm{~kg}$.

$$
\begin{aligned}
\frac{\rho_{m}}{\rho_{w}} & =729 \frac{M_{m}}{M_{w}} \\
& =729\left(\frac{412.980 \mathrm{~kg}}{66076.5 \mathrm{~kg}}\right) \\
& =4.556
\end{aligned}
$$

Table 4.1: Results of the free scaled model optimization.

\begin{tabular}{|c|c|c|c|}
\hline Property & Scaled target model value & Optimized model value & \% difference \\
\hline $\begin{array}{c}\text { Elastic mode } \\
1 \omega(\mathrm{Hz})\end{array}$ & 2.223 & 2.199 & $-1.080 \%$ \\
\hline $\begin{array}{c}\text { Elastic mode } \\
2 \omega(\mathrm{Hz})\end{array}$ & 2.798 & 2.816 & $0.643 \%$ \\
\hline $\begin{array}{c}\text { Elastic mode } \\
3 \omega(\mathrm{Hz})\end{array}$ & 3.740 & 3.854 & $3.048 \%$ \\
\hline $\begin{array}{c}\text { Elastic mode } \\
4 \omega(\mathrm{Hz})\end{array}$ & 5.015 & 5.000 & $-0.299 \%$ \\
\hline $\begin{array}{c}\text { Elastic mode } \\
5 \omega(\mathrm{Hz})\end{array}$ & 6.043 & 6.320 & $4.584 \%$ \\
\hline$x_{c g}(\mathrm{~mm})$ & 1240.49 & 1182.44 & $-4.680 \%$ \\
\hline$y_{c g}(\mathrm{~mm})$ & $\sim 0.0$ & $\sim 0.0$ & $\mathrm{~N} / \mathrm{A}$ \\
\hline$z_{c g}(\mathrm{~mm})$ & 113.95 & 108.48 & $-4.800 \%$ \\
\hline$I_{y y}\left(\mathrm{~kg} \cdot \mathrm{m}^{2}\right)$ & 173.5 & 169.2 & $-2.428 \%$ \\
\hline
\end{tabular}

Figures 4.3 through 4.7 show the aeroelastic paneling mode shapes generated from the target and scaled models. Qualitative similarity is seen in each of the shapes. Before applying constraints to match the center of gravity location and pitch inertia, large differences in rigid body pitch rotation were visible when comparing the first, fourth, and fifth elastic modes. The application of these constraints has alleviated most of this discrepancy in the first elastic mode, but some extraneous rotation is still visible in the fourth and fifth modes. 


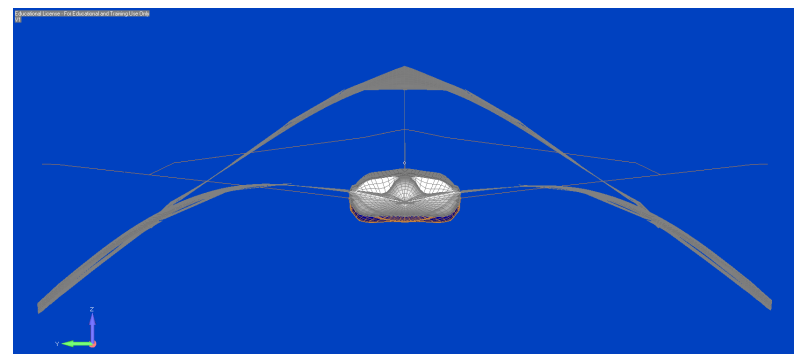

(a) Target first elastic panel mode shape.

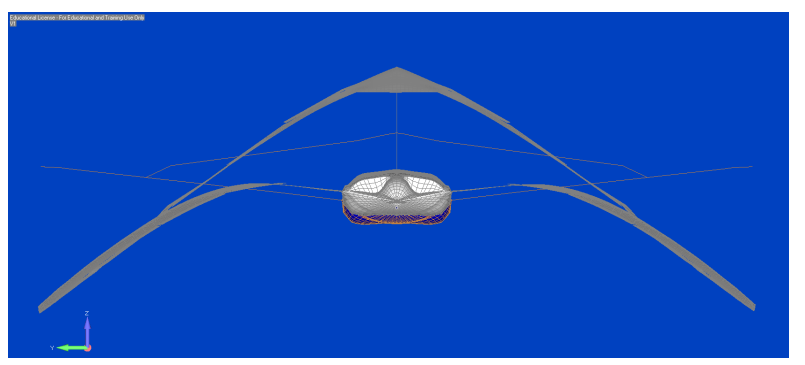

(b) Scaled first elastic panel mode shape.

Figure 4.3: First elastic mode shape comparison.

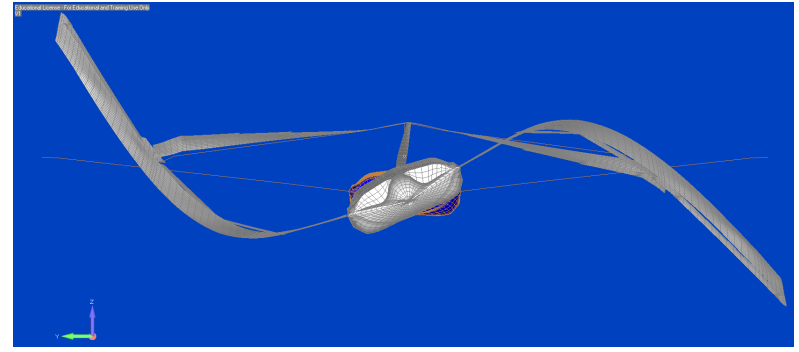

(a) Target second elastic panel mode shape.

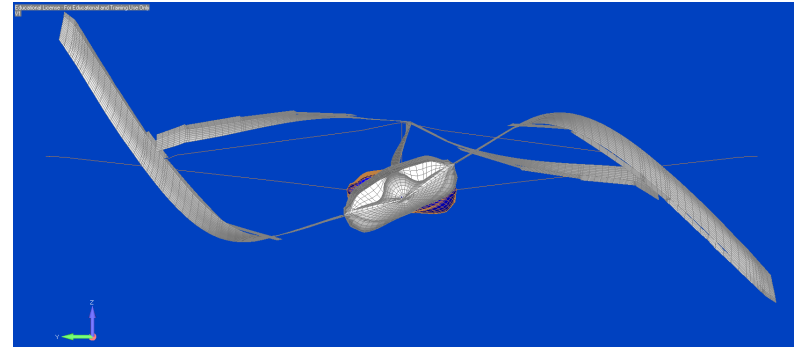

(b) Scaled second elastic panel mode shape.

Figure 4.4: Second elastic mode shape comparison.

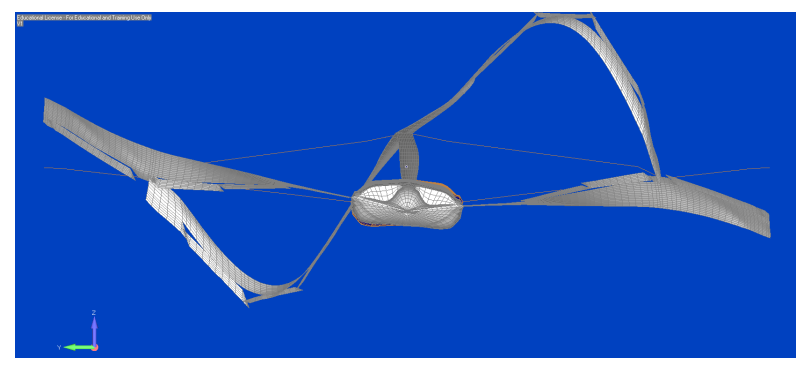

(a) Target third elastic panel mode shape.

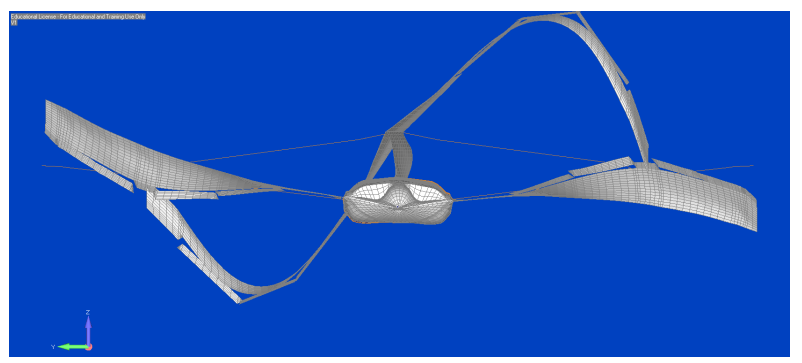

(b) Scaled third elastic panel mode shape.

Figure 4.5: Third elastic mode shape comparison. 


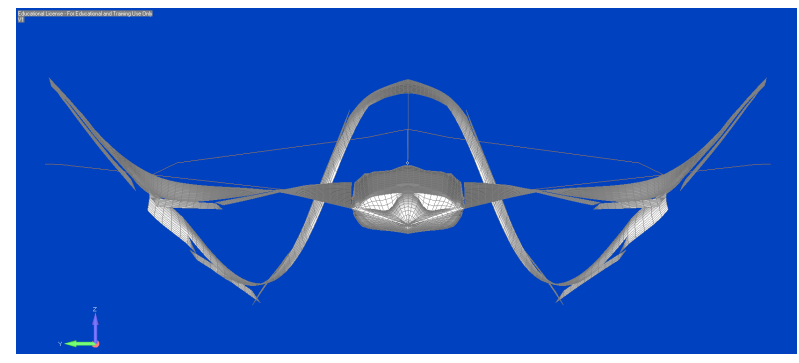

(a) Target fourth elastic panel mode shape.

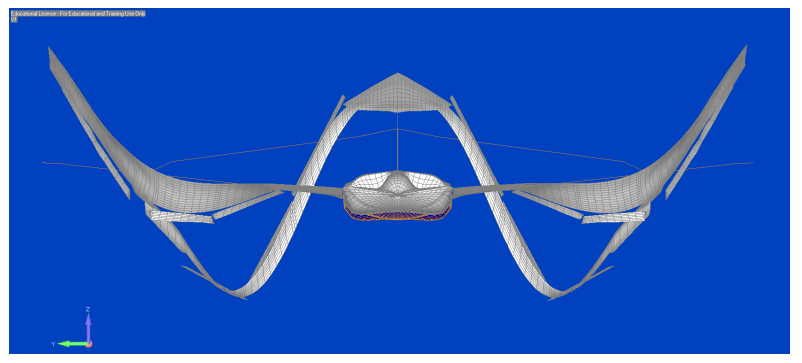

(b) Scaled fourth elastic panel mode shape.

Figure 4.6: Fourth elastic mode shape comparison.

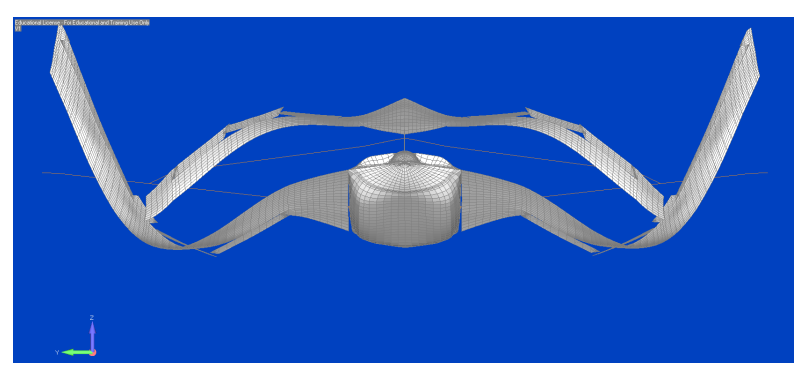

(a) Target fifth elastic panel mode shape.

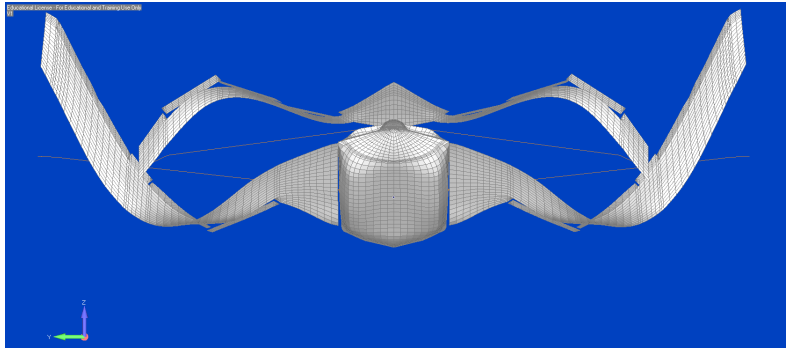

(b) Scaled fifth elastic panel mode shape.

Figure 4.7: Fifth elastic mode shape comparison.

\subsubsection{Constrained Vibration Case}

Table 4.2 shows the results of the constrained vibration optimization routine. With no constraints on the total mass, center of gravity location, or pitch inertia, the first six natural frequencies were successfully matched to within $\pm 2.6 \%$. The first three frequencies were not able to be matched as closely as desired. Excluding the center of gravity and pitch inertia constraints was only beneficial by nature of the boundary conditions. As with the free vibration case, Equation 3.24 was used to set the density ratio using the unconstrained total mass of the scaled model, approximately $363 \mathrm{~kg}$. 


$$
\begin{aligned}
\frac{\rho_{m}}{\rho_{w}} & =729 \frac{M_{m}}{M_{w}} \\
& =729\left(\frac{362.705 \mathrm{~kg}}{66076.5 \mathrm{~kg}}\right) \\
& =4.002
\end{aligned}
$$

Table 4.2: Results of the constrained scaled model optimization.

\begin{tabular}{|c|c|c|c|}
\hline Property & Scaled target model value & Optimized model value & \% difference \\
\hline Mode $1 \omega(\mathrm{Hz})$ & 1.239 & 1.207 & $-2.583 \%$ \\
\hline Mode $2 \omega(\mathrm{Hz})$ & 2.098 & 2.150 & $2.479 \%$ \\
\hline Mode $3 \omega(\mathrm{Hz})$ & 2.538 & 2.601 & $2.482 \%$ \\
\hline Mode $4 \omega(\mathrm{Hz})$ & 3.704 & 3.736 & $0.864 \%$ \\
\hline Mode $5 \omega(\mathrm{Hz})$ & 4.929 & 4.921 & $-0.162 \%$ \\
\hline Mode $6 \omega(\mathrm{Hz})$ & 5.110 & 5.130 & $0.391 \%$ \\
\hline
\end{tabular}

Figures 4.8 through 4.13 show the aeroelastic paneling mode shapes generated from the target and scaled models. Very good qualitative similarity is seen in each of the shapes, but some noticeable differences are present. There is a large discrepancy in the curvature of the aft wing in the third mode shape. This problem seems to be unrelated to the difficulty in matching the inertias and might be alleviated by changing the objective function weight $W_{3}$. There is also significant difference in the local pitch angle of the tail boom strake in the sixth mode shape. From the horizontal plane, the target mode shape shows $9.03^{\circ}$ pitch down in the strake while the scaled shape shows $14.08^{\circ}$ pitch down. This difference demonstrates the importance of matching the pitch inertia, even in the constrained vibration case. While 
the global rigid body pitch problem was removed using the boundary conditions, local rigid pitch effects such as those in the tail boom strake are still a concern. Adding pitch inertia constraints to this case may alleviate some of these differences.

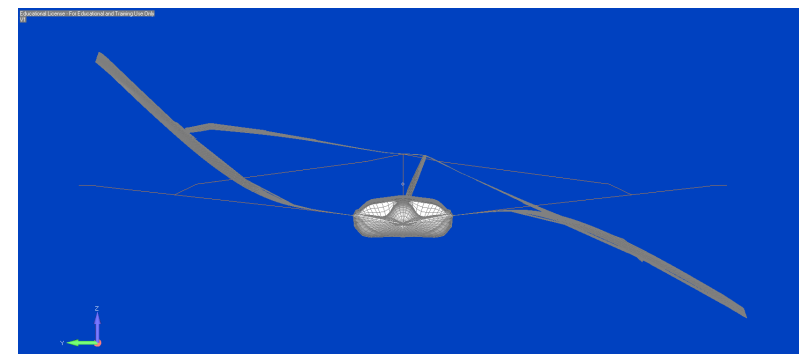

(a) Target first panel mode shape.

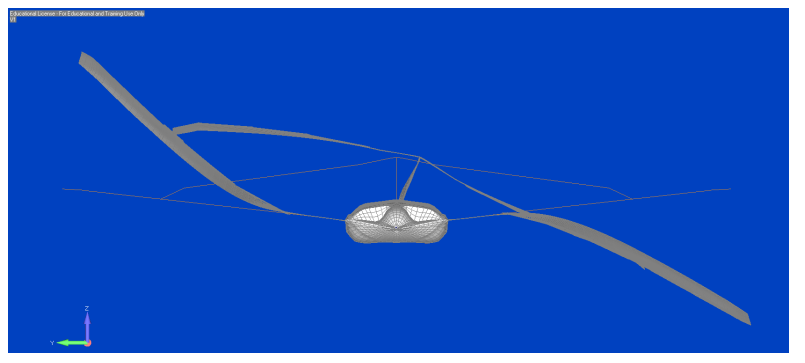

(b) Scaled first panel mode shape.

Figure 4.8: First constrained mode shape comparison.

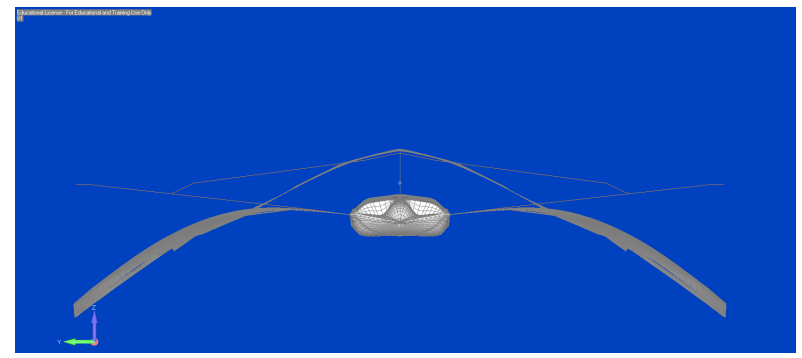

(a) Target second panel mode shape.

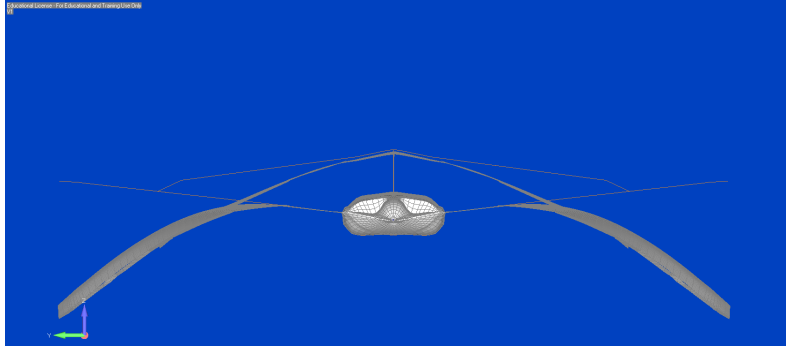

(b) Scaled second panel mode shape.

Figure 4.9: Second constrained mode shape comparison.

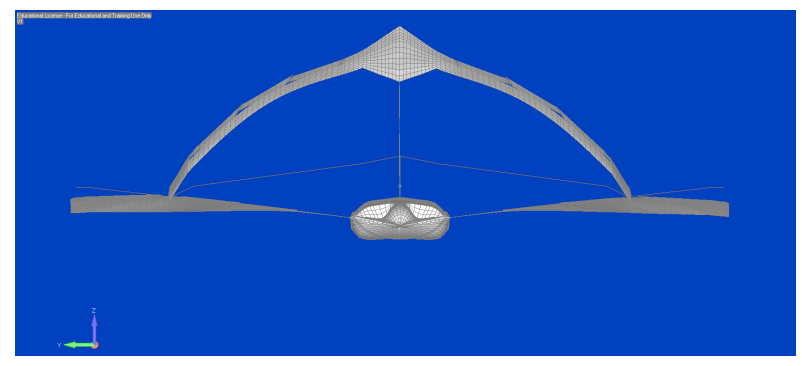

(a) Target third panel mode shape.

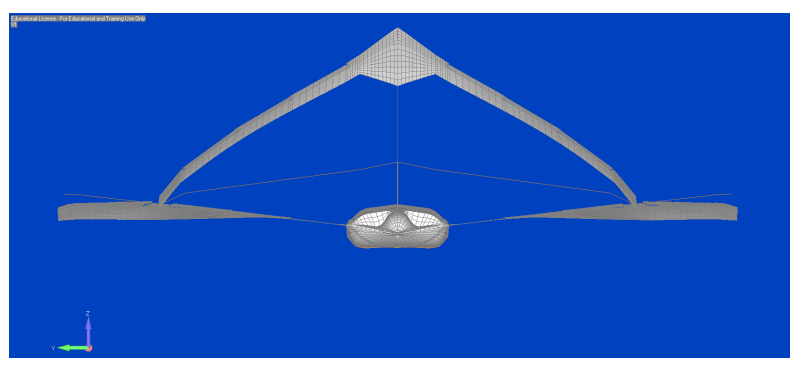

(b) Scaled third panel mode shape.

Figure 4.10: Third constrained mode shape comparison. 


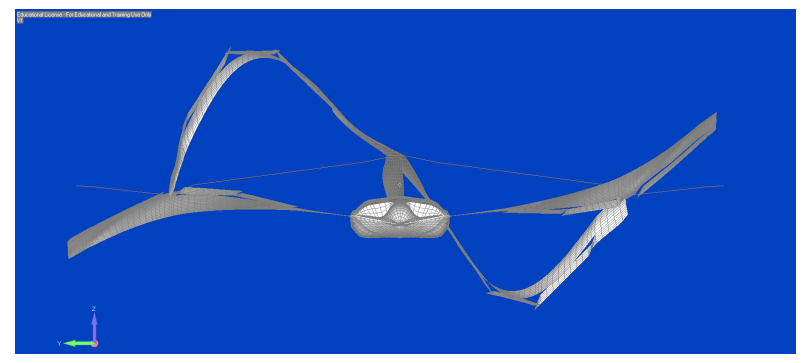

(a) Target fourth panel mode shape.

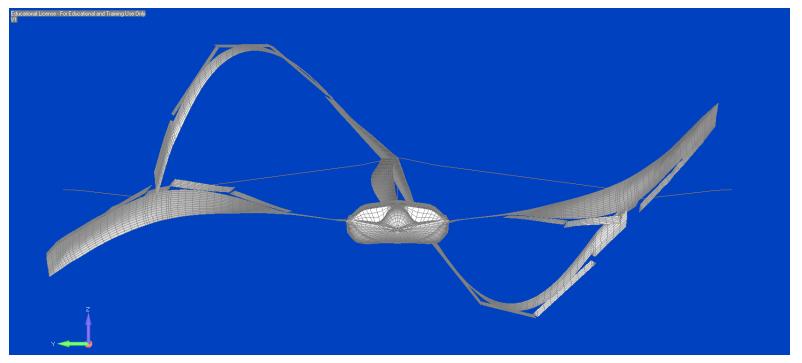

(b) Scaled fourth panel mode shape.

Figure 4.11: Fourth constrained mode shape comparison.

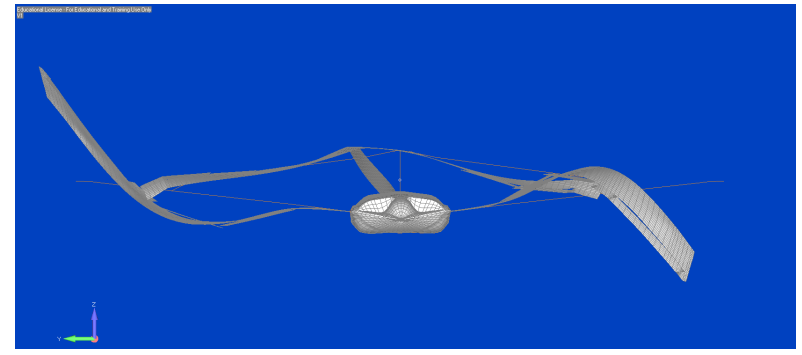

(a) Target fifth panel mode shape.

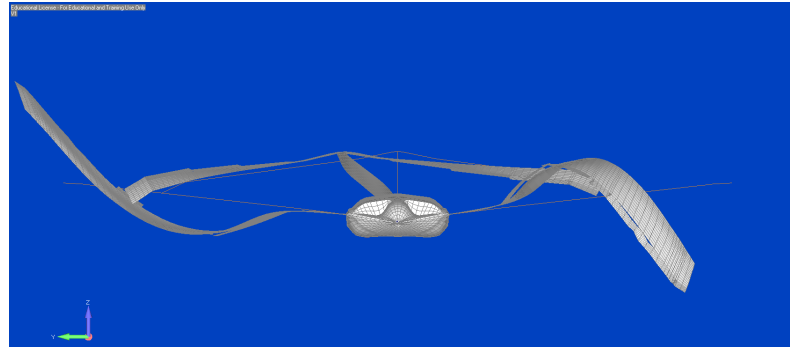

(b) Scaled fifth panel mode shape.

Figure 4.12: Fifth constrained mode shape comparison.

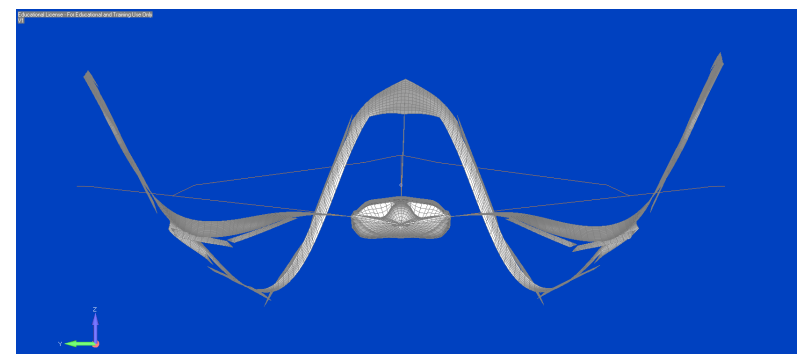

(a) Target sixth panel mode shape.

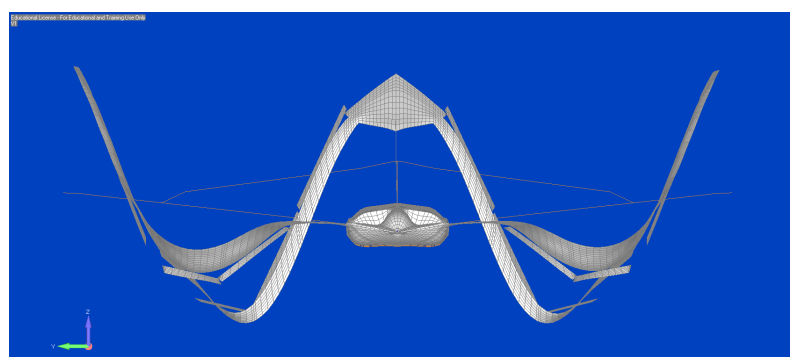

(b) Scaled sixth panel mode shape.

Figure 4.13: Sixth constrained mode shape comparison. 


\section{Chapter 5}

\section{Aeroelastic Response Verification}

A flutter analysis was conducted on the optimized scaled models using ZONA's ZAERO software in order to verify the scaling procedure. The ratios calculated in equations 4.2 and 4.3 were used to set the flight densities of the models. Table 5.1 shows the chosen densities and corresponding altitudes. Infinite plate splines were used to transfer deformations and loads between the structural finite element model and the aerodynamic panel model.

Table 5.1: Flight densities and altitudes for aeroelastic response verification.

\begin{tabular}{|c|c|c|c|}
\hline & Scaled model & Target free case & Target constrained case \\
\hline $\begin{array}{c}\text { Flight density } \\
\left(\mathrm{kg} / \mathrm{m}^{3}\right)\end{array}$ & 1.225 & 0.269 & 0.306 \\
\hline Altitude $(\mathrm{m})$ & Sea level & $\sim 13,000$ & $\sim 12,000$ \\
\hline
\end{tabular}




\subsection{Free Vibration Aeroelastic Results}

Figures 5.1 and 5.2 show the aeroelastic damping and frequency plots, respectively, of the free flutter analysis. The data from the scaled model was normalized to the full-scale target regime.

Decent similarity is seen in all of the aeroelastic modes except mode three on the damping plot up to a scaled velocity of approximately 500 knots. Past 600 knots, aeroelastic modes one and four coalesce in a fashion not seen in the target model.

Very good similarity is seen in all of the modes on the frequency plot up to a scaled velocity of approximately 300 knots. Past this point, scaled mode four begins to drift away from the target until it coalesces with scaled mode one. The original difference in matching of the third and fifth elastic frequencies within the optimization process is also evident.

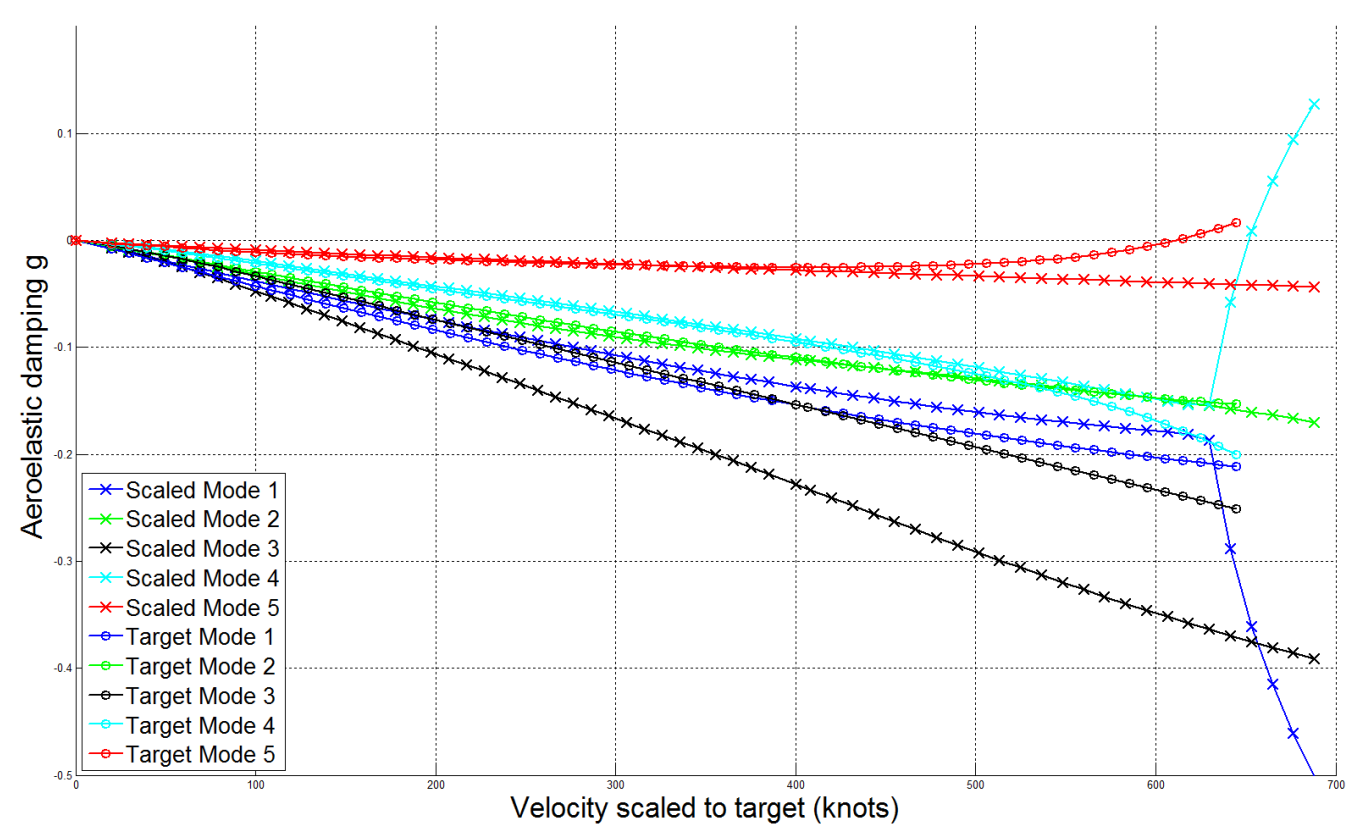

Figure 5.1: Aeroelastic damping from the free flutter analysis. 


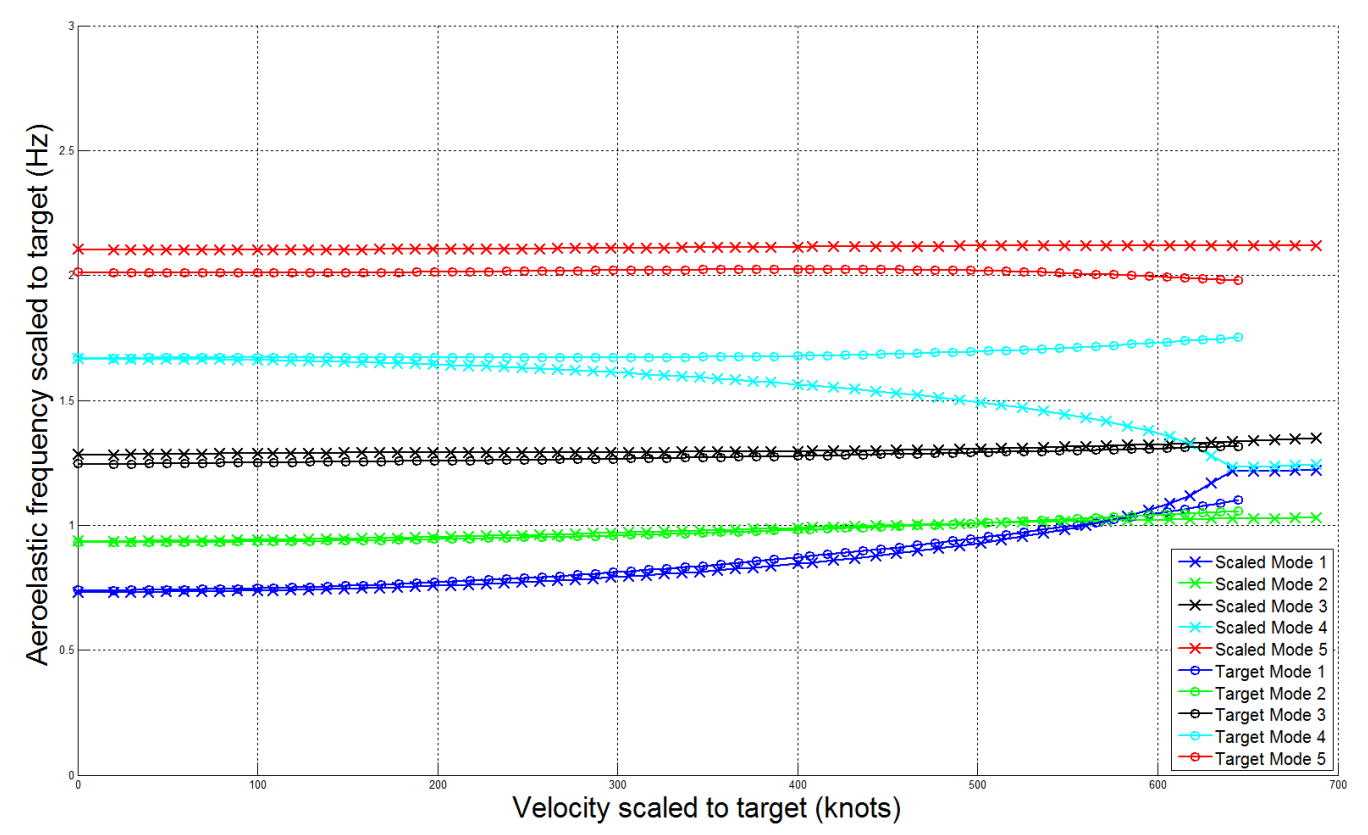

Figure 5.2: Aeroelastic frequency from the free flutter analysis.

Table 5.2 compares the critical free flutter mode found using each model. Though the scaled flutter velocities show reasonable agreement, they occur in different aeroelastic modes. The flutter frequencies are also not in agreement. Different flutter modes are being captured, as seen in Figure 5.3. Modal participation factors show that the target flutter mode is mainly comprised of the fifth elastic mode coupled with the fourth mode. The scaled flutter mode is mainly comprised of the fourth elastic mode coupled with the first mode. Table 5.3 shows the modal participation factors for the target and scaled free flutter modes. The factors are normalized such that the primary mode has $100 \%$ participation.

The discrepancies in the aeroelastic response of the free vibration case are most likely attributed to the remaining pitch rotation differences between the models. The fourth and fifth elastic modes still show significant pitch differences, shapes that are important to the 
target critical flutter response. Better similarity should also be achieved by matching the third and fifth elastic frequencies closer to their targets.

Table 5.2: Free flutter results.

\begin{tabular}{|c|c|c|c|}
\hline Property & Target value & Scaled optimized value & \% difference \\
\hline Flutter mode & 5 & 4 & N/A \\
\hline Velocity $(\mathrm{knots})$ & 610.670 & 651.619 & $6.706 \%$ \\
\hline Frequency $(\mathrm{Hz})$ & 1.992 & 1.235 & $-38.002 \%$ \\
\hline
\end{tabular}

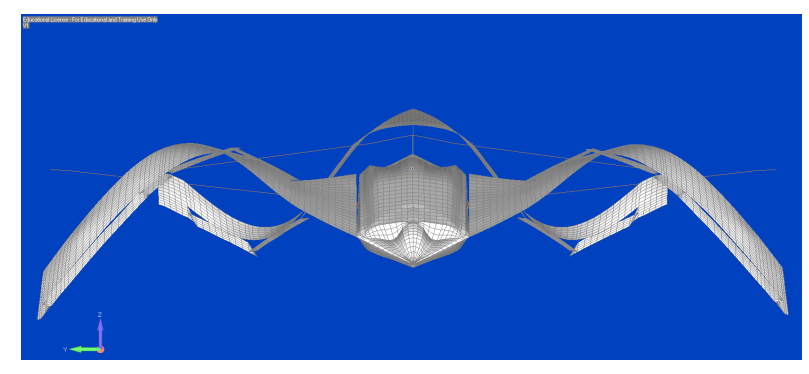

(a) Target flutter panel mode shape.

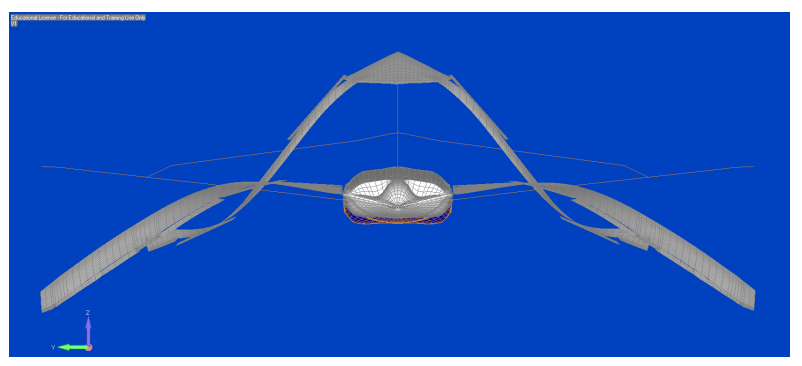

(b) Scaled flutter panel mode shape.

Figure 5.3: Free flutter mode shape comparison.

Table 5.3: Free flutter modal participation factors.

\begin{tabular}{|l|c|c|}
\hline & Target partcipation & Scaled participation \\
\hline Mode 1 & $4.508 \%$ & $83.671 \%$ \\
\hline Mode 2 & $0.008 \%$ & $0.010 \%$ \\
\hline Mode 3 & $0.001 \%$ & $0.013 \%$ \\
\hline Mode 4 & $50.038 \%$ & $100 \%$ \\
\hline Mode 5 & $100 \%$ & $6.368 \%$ \\
\hline
\end{tabular}

\subsection{Constrained Vibration Aeroelastic Results}

Figures 5.4 and 5.5 show the aeroelastic damping and frequency plots, respectively, of the constrained flutter analysis. The data from the scaled model was normalized to the full-scale 
target regime.

Decent similarity is seen in all of the aeroelastic modes except mode six on the damping plot up to a scaled velocity of approximately 500 knots. The damping in mode 6 never matches as closely as the other modes, but the scaled and target values do trend in the same direction.

Decent similarity is seen in all of the aeroelastic modes on the frequency plot through nearly the entire velocity profile. Modes three and six show the greatest difference at higher velocities, but these modes always trend in the same fashion.

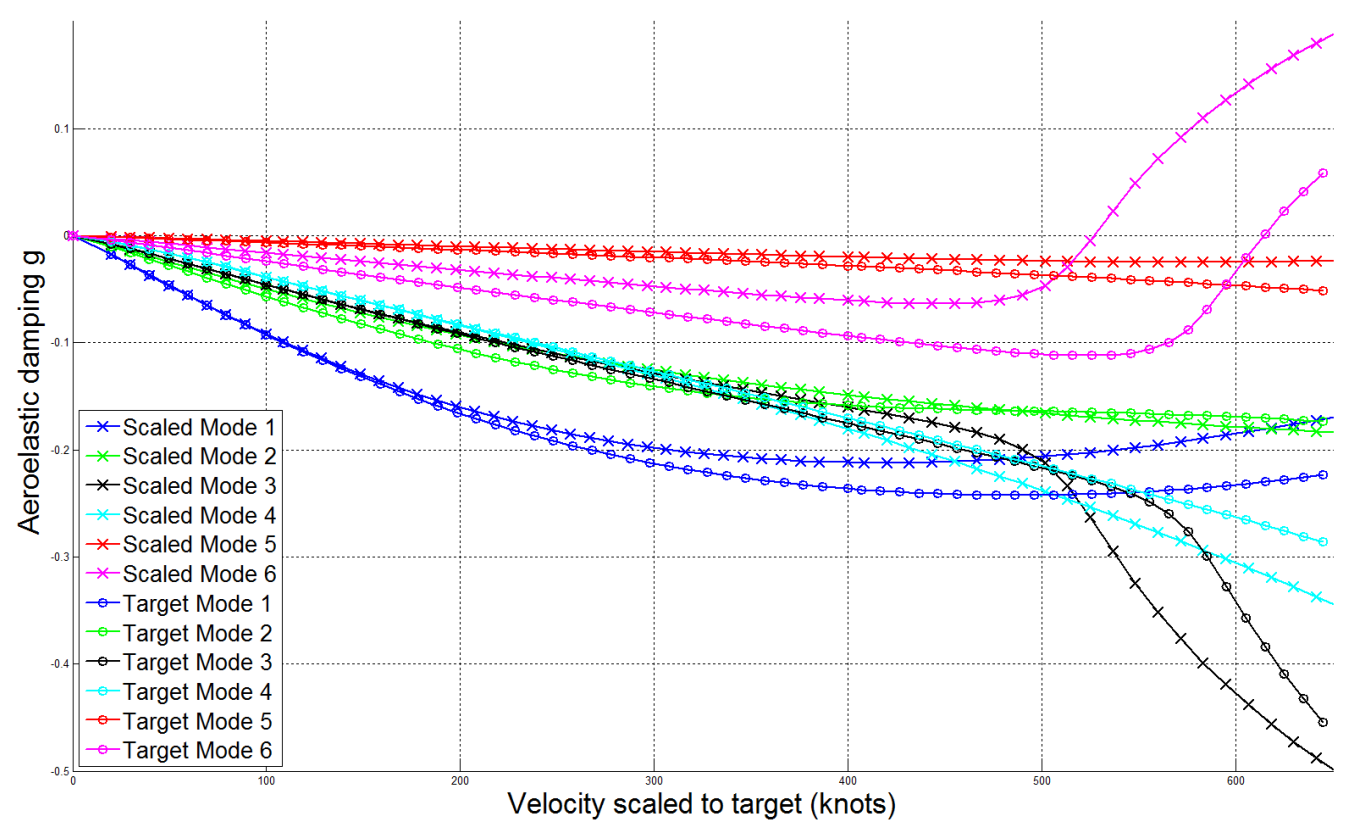

Figure 5.4: Aeroelastic damping from the constrained flutter analysis. 


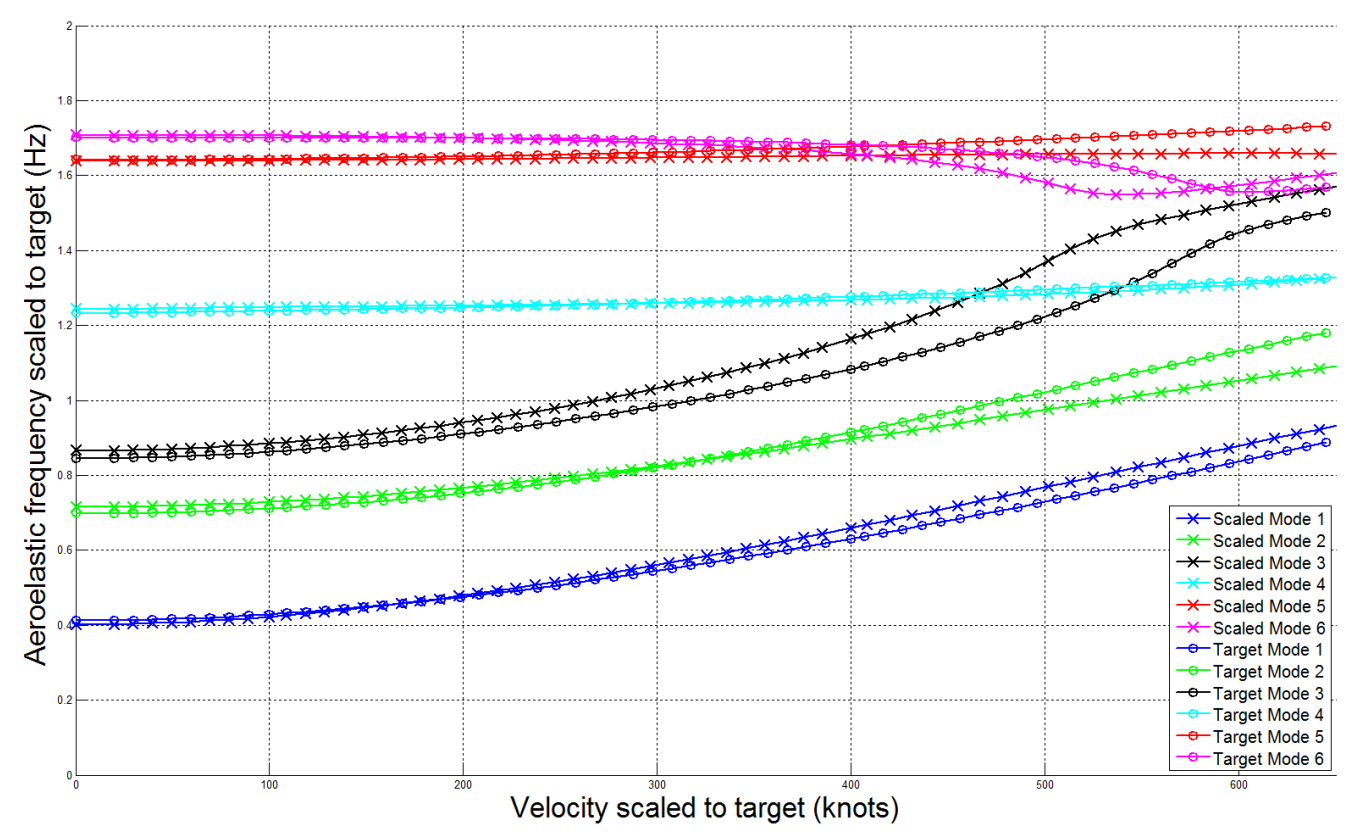

Figure 5.5: Aeroelastic frequency from the constrained flutter analysis.

Table 5.4 compares the critical constrained flutter mode found using each model. The flutter velocities are not as close as desired in order to deem the scaling procedure a complete success. Unlike the free vibration case, however, the same flutter mode is being captured as seen in Figure 5.6. The flutter frequencies also match very well. Modal participation factors show that this flutter mechanism is mainly comprised of the third structural mode coupled with the sixth mode. Table 5.5 shows the modal participation factors for the target and scaled constrained flutter modes.

The initial differences in the third and sixth mode shapes are evident in the flutter mode shapes. This is to be expected since the flutter mechanism is mainly comprised of these two modes. The aft wing shows a slightly different curvature between the target and scaled shapes, and the tail boom strake is again pitched differently. The strake is pitched down 
$13.64^{\circ}$ in the target shape and $16.28^{\circ}$ in the scaled shape. The incorrectly scaled inertia in the strake seems to be causing the entire tail boom to not deform as expected. Adding inertia constraints to the optimization routine of this case may alleviate the problem.

Table 5.4: Constrained flutter results.

\begin{tabular}{|c|c|c|c|}
\hline Property & Target value & Scaled optimized value & \% difference \\
\hline Flutter mode & 6 & 6 & N/A \\
\hline Velocity $(\mathrm{knots})$ & 614.218 & 526.744 & $-14.242 \%$ \\
\hline Frequency $(\mathrm{Hz})$ & 1.557 & 1.553 & $-0.257 \%$ \\
\hline
\end{tabular}

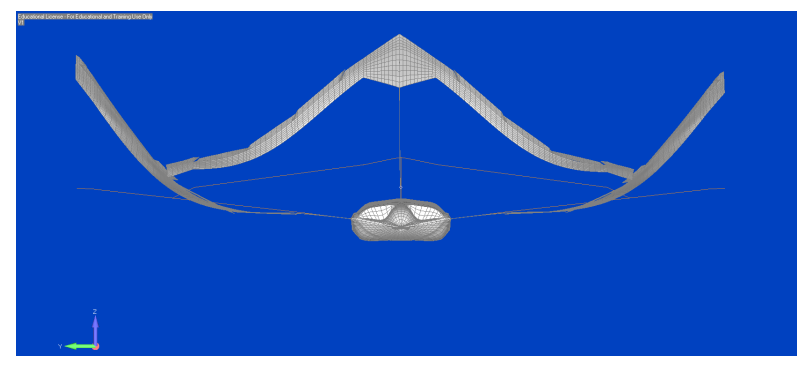

(a) Target flutter panel mode shape.

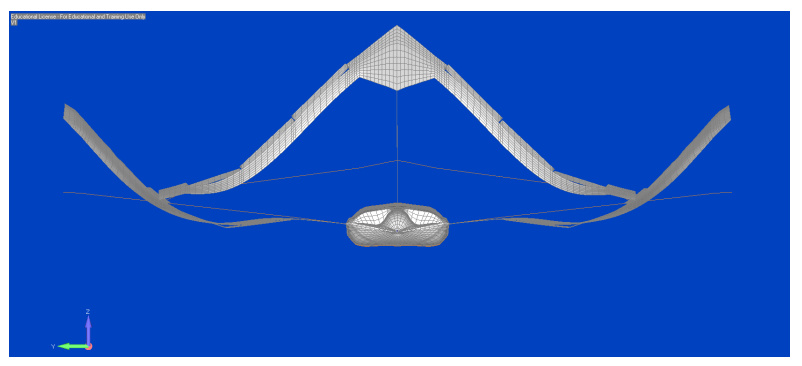

(b) Scaled flutter panel mode shape.

Figure 5.6: Constrained flutter mode shape comparison.

Table 5.5: Constrained flutter modal participation factors.

\begin{tabular}{|l|c|c|}
\hline & Target partcipation & Scaled participation \\
\hline Mode 1 & $0.018 \%$ & $0.001 \%$ \\
\hline Mode 2 & $1.908 \%$ & $4.881 \%$ \\
\hline Mode 3 & $100 \%$ & $100 \%$ \\
\hline Mode 4 & $0.006 \%$ & $0.005 \%$ \\
\hline Mode 5 & $0.316 \%$ & $0.012 \%$ \\
\hline Mode 6 & $67.758 \%$ & $58.298 \%$ \\
\hline
\end{tabular}




\section{Chapter 6}

\section{Conclusions and Recommendations}

\subsection{Conclusions}

Two modal optimizations were performed on a $1 / 9^{\text {th }}$ span RPV finite element model, yielding partially aeroelastically scaled designs. The aeroelastic response showed good similarity in most modes of both the free and constrained vibration cases. In the free vibration case, a scaled flutter mode was captured that was near the target flutter velocity but not frequency. However, this scaled flutter mode occurred in a different aeroelastic mode than the target and was mainly comprised of different structural modes. The correct flutter mode was captured in the constrained vibration case. Although the flutter velocity in this case did not match as closely as desired, the flutter frequency was nearly identical to the target value.

The results presented show that aeroelastic scaling through a modal optimization is 
possible. The methodology presented by Bond et al. ${ }^{17}$ has been extended to a built-up model representative of a flight test vehicle including rigid body degrees of freedom. The error in the aeroelastic response is most likely due to the remaining difference in the scaled mode shapes and frequencies, especially the extraneous rigid body rotation still present in the fourth and fifth elastic modes of the free vibration case. Bond demonstrated the importance in accurately matching the mode shapes to produce a correctly scaled design, and this importance is evident in the presented results. The presented work has also demonstrated the need to correctly match the scaled center of gravity location and inertia. Without these mass property constraints, the free vibration case produced poorly matching mode shapes with extraneous rigid body pitch rotations. By removing the majority of the inertia matching problems in the constrained vibration case, much better results were produced.

During the scaling procedure, manufacturability was neglected in order to verify the aeroelastic scaling procedure with as much design freedom as possible. Thus, the resulting optimal designs presented would be impossible to build and test. The accuracy in the ply layers needed to attain these results would be impossible to reproduce. Differences in ply thicknesses of some thin layers of the optimal designs were on the order of $1 / 1000^{\text {th }} \mathrm{mm}$, a resolution not possible with the current wet layup technique. The uncertainty in the resin control of the manufacturing process would likely produce a vehicle that was not representative of the designed finite element model. In addition, the high final mass of the vehicles would require much larger propulsion systems than those currently implemented. The flight test RPV was designed for take off weights in the range of approximately $60-90 \mathrm{~kg}$, and each 
of the vehicle's two jet engines was rated for approximately $20 \mathrm{~kg}$ of thrust. ${ }^{4}$ The optimal designs presented in this work were on the order of $400 \mathrm{~kg}$. Thus, a propulsion system roughly four times as powerful would be needed to fly the designed vehicles. With these concerns in mind, it can be concluded that it would be infeasible to design an aeroelastically scaled flight test vehicle using the as built $1 / 9^{\text {th }}$ span RPV as a starting point. The same carbon fiber ply layup manufacturing technique is not suited for the scaled design, and an entirely new structure would need to be designed.

\subsection{Recommendations}

Better matching the target frequencies and mode shapes is required for producing a better scaled aeroelastic response. This section outlines some recommendations on how to achieve this better matching modal response while employing the same overall scaling technique and methodology.

The presented results have showed that matching the scaled center of gravity location and pitch inertia is critically important to matching the mode shapes and thus the aeroelastic response. The free vibration case employed a $\pm 5 \%$ constraint on these mass properties, so more stringent constraints could potentially produce better mode shapes. Some pitch concerns remained in the constrained vibration case, especially in the tail boom strake. Thus, adding inertia constraints to this case could also produce better mode shapes.

In order to get specific mode shapes and frequencies to match closer, the weights $W_{i}$ in 
the objective function and the frequency constraints could be changed. A higher weight and more stringent frequency constraint could cause one mode to look better, likely at the cost of other modes. Increasing $W_{3}$ and $W_{6}$ in the constrained vibration case could potentially produce a better aeroelastic response since these were the worst matching modes and those most important to the flutter mechanism.

The objective function itself could be improved by adding more matching nodes and more degrees of freedom. Only translational degrees of freedom have been used in this work. Adding rotational freedoms may produce better mode shapes. In addition, wing twist could be better matched by calculating and matching normalized slopes between the leading and trailing edges. 


\section{References}

[1] Wolkovitch, J., "The Joined Wing: An Overview," Journal of Aircraft, Vol. 23, No. 3, 1986, pp. 161-178.

[2] Gallman, J. W. and Kroo, I. M., "Structural Optimization for Joined-Wing Synthesis," Journal of Aircraft, Vol. 33, No. 1, Jan. 1996, pp. 214-223.

[3] Blair, M., Canfield, R. A., and Roberts, R. W., "Joined-Wing Aeroelastic Design with Geometric Nonlinearity," Journal of Aircraft, Vol. 42, No. 4, July 2005, pp. 832-848.

[4] Aarons, T., Development and Implementation of a Flight Test Program for a Geometrically Scaled Joined Wing SensorCraft Remotely Piloted Vehicle, Master's thesis, Virginia Polytechnic Institute and State University, 2011.

[5] Richards, J., Aarons, T., Suleman, A., Canfield, R. A., Woolsey, C., Lindsley, N., and Blair, M., "Design for Flight Test of a Scaled Joined Wing SensorCraft," 52nd AIAA/ASME/ASCE/AHS/ASC Structures, Structural Dynamics \& Materials Conference, No. AIAA 2011-2011, 2011. 
[6] Macagno, E. O., "Historico-critical Review of Dimensional Analysis," Journal of the Franklin Institute, Vol. 292, No. 6, 1971.

[7] Vaschy, A., "Sur les lois de similitude en physique," Annales Télégraphiques, Vol. 19, 1892, pp. 25-28.

[8] Buckingham, E., "On Physically Similar Systems," Physical Review, Vol. 4, No. 4, 1914, pp. $345-376$.

[9] Bisplinghoff, R. L., Ashley, H., and Halfman, R. L., Aeroelasticity, Addison-Wesley, Cambridge, MA, 1955.

[10] Molyneux, W., “Aeroelastic Modelling," RAE Technical Note, No. Structures 353, 1964.

[11] Wolowicz, C. H., Bowman, J. S., and Gilbert, W. P., "Similitude Requirements and Scaling Relationships as Applied to Model Testing," NASA Technical Paper, No. 1435, 1979.

[12] Friedmann, P. P. and Presente, E., "Active Control of Flutter in Compressible Flow and Its Aeroelastic Scaling," Journal of Guidance, Control, and Dynamics, Vol. 24, No. 1, Jan. 2001, pp. 167-175.

[13] Pototzky, A. S., "Scaling Laws Applied to a Modal Formulation of the Aeroservoelastic Equations," 43rd AIAA/ASME/ASCE/AHS/ASC Structures, Structural Dynamics, \& Materials Conference, No. AIAA 2002-1598, 2002. 
[14] French, M. and Eastep, F., "Aeroelastic Model Design Using Parameter Identification," Journal of Aircraft, Vol. 33, No. 1, 1996.

[15] Richards, J., Suleman, A., Canfield, R. A., and Blair, M., "Design of a Scaled RPV for Investigation of Gust Response of Joined-Wing Sensorcraft," 50th AIAA/ASME/ASCE/AHS/ASC Structures, Structural Dynamics, $\&$ Materials Conference, No. AIAA 2009-2218, 2009.

[16] Pereira, P., Almeida, L., Suleman, A., Bond, V., Canfield, R. A., and Blair, M., "Aeroelastic Scaling and Optimization of a Joined-Wing Aircraft Concept," 48th AIAA/ASME/ASCE/AHS/ASC Structures, Structural Dynamics, \&6 Materials Conference, No. AIAA 2007-1889, 2007.

[17] Bond, V. L., Canfield, R. A., Suleman, A., and Blair, M., "Aeroelastic Scaling of a Joined Wing for Nonlinear Geometric Stiffness," AIAA Journal, Vol. 50, No. 3, March 2012, pp. 513-522.

[18] Ricciardi, A. P., Canfield, R. A., Patil, M. J., and Lindsley, N., "Nonlinear Aeroelastic Scaling of a Joined-Wing Aircraft," 53rd AIAA/ASME/ASCE/AHS/ASC Structures, Structural Dynamics \& Materials Conference, No. AIAA 2012-1454, 2012.

[19] Wan, Z. and Cesnik, C. E., "Geometrically Nonlinear Aeroelastic Scaling for Very Flexible Aircraft," 54th AIAA/ASME/ASCE/AHS/ASC Structures, Structural Dynamics, E Materials Conference, No. AIAA 2013-1894, 2013. 
[20] Richards, J., Garnand-Royo, J., Ricciardi, A., Suleman, A., Canfield, R. A., and Woolsey, C., "Design and Evaluation of Aeroelastically Tuned Joined-Wing SensorCraft Flight Test Article," 54th AIAA/ASME/ASCE/AHS/ASC Structures, Structural Dynamics \& Materials Conference, No. AIAA 2013-1786, 2013. 
Appendices 


\section{Appendix A}

\section{Finite Element Model Conversion}

The initial design point for the modal optimization problem presented in this thesis was

a high fidelity finite element model of the $1 / 9^{t h}$ span geometrically scaled RPV provided by Richards et al. ${ }^{5}$ The original finite element model was coded in ANSYS. This appendix details the conversion process performed to create an acceptably equivalent model in Nastran. The converted Nastran model was used in the aeroelastic scaling procedure of this thesis.

\section{A.1 Initial ANSYS Conversion}

The original finite element model was coded within the ANSYS Classic environment. To begin the conversion process, the model was exported to an archive file using the following command from the ANSYS command line: 


$$
\text { [ cdwrite, db, 'file', 'cdb' ] }
$$

This command produced a .cdb file that was imported into Femap, a Nastran pre/post processor program. This initial import into Femap only correctly converted certain portions of the original ANSYS model. The items correctly converted included:

1. Node locations and numbering

2. Element location, numbering, and material coordinate system orientation

3. Beam and rod properties

4. Concentrated mass properties, locations, and rigid connections to the model

Items incorrectly imported included:

1. Some material properties

2. Ply element properties

3. Connections between separate pieces of the model (such as the wings to the fuselage)

Each of these items was manually converted from the original ANSYS model.

\section{A.2 Fixing the Material Properties}

Most of the material properties were correctly imported by Femap during the initial conversion, but some corrections in formatting were required. Material properties were defined 
in the ANSYS .cdb file using the MPDATA and MPTEMP commands. Using the ANSYS Command Reference and Nastran Quick Reference Guide, individual material properties were converted into Nastran MAT8 cards. MAT8 cards define orthotropic materials in Nastran. Isotropic materials from ANSYS could also be written to MAT8 cards by defining the same properties in each direction. Temperature dependent properties were written to Nastran MAT4 cards.

\section{A.3 Fixing the Ply Element Properties}

None of the ply layer element properties were imported correctly during the initial conversion. Ply properties were defined in the ANSYS .cdb file using the $E B L O C K$ and $S E C^{*}$ commands. Custom written MATLAB scripts were used to read the data from these commands and write them to Nastran PCOMP cards. PCOMP cards define ply layer element properties in Nastran.

Each line of numbers in the EBLOCK command represented a single element within ANSYS. The first large section of the EBLOCK command corresponded to the ply elements. The pertinent fields of each line were defined as follows. Fields 1-3 denoted the ANSYS "property" number. Field 4 denoted the section number of a corresponding SECTYPE command. The SECTYPE commands contained the actual information about the ply layup including number of plys, ply thicknesses, ply materials, and ply angles. Field 5 denoted the element coordinate system number (already correctly read). Field 11 denoted the element 
number (already correctly read). Fields 12-19 denoted the node numbers of the element (already correctly read).

A custom MATLAB script was used to assign each element a property number related to fields 1-3. Each ANSYS defined "property" generally consisted of the same SECTYPE. Thus, each ANSYS "property" received a corresponding Nastran PCOMP card numbered x001 where x was the "property" number. Should an ANSYS "property" consist of more than one SECTYPE, the ones place of the PCOMP card was increased. For example, PCOMP 1003 referred to the third SECTYPE of the first ANSYS "property." Approximately 890 types of PCOMP property cards were defined this way. Some exceptions were made to account for the changing ANSYS "property" numbers. One exception to this rule was ANSYS "property" 19, which consisted of elements within the body of the fuselage. Every one of these elements had a unique SECTYPE, so a unique PCOMP card was written for each element. Elements within the fuselage received PCOMP cards starting at number 900001.

The ANSYS SECTYPE commands contained the actual ply information written to the Nastran PCOMP cards. The SECTYPE command defined the section number, referenced in field 4 of an EBLOCK line. Following this, a SECOFFSET command defined the reference plane of the section. Next, the SECBLOCK command defined the number of layers $n$ in the ply section. The following $n$ lines defined the ply thickness, ply material, and ply angle of the particular layer. The SECCONTROLS command was not used in the conversion.

The combination of EBLOCK and $S E C^{*}$ commands was used to link each Nastran element with a PCOMP card containing the ply layup information. 


\section{A.4 Fixing the Model Connections}

Large pieces of the vehicle such as each of the wings, the control surfaces, and the fuselage were not connected through traditional elements within the original ANSYS model. These individual pieces were "glued" together using ANSYS contact elements. Unfortunately, no easily implementable equivalent of these elements exists within the version of MSC.Nastran used in this thesis. MSC.Nastran provides BCBODY and BSURF cards to define contact regions, but these commands were never successfully implemented into the model. A simple and effective solution was to tie the parts together using Nastran RBE2 elements. These rigid bar elements were defined within overlapping areas of the connection regions. If nodes were within a certain defined distance of each other, a rigid bar element was placed between them, forcing the nodes to deflect and rotate the same amount.

Using rigid elements between nodes sets the "stiffness" within the connection regions. The tied nodes cannot move away from each other, creating a very stiff connection. To allow for some design freedom with the connection stiffness, Nastran CROD elements could be defined between the nodes with corresponding PROD properties. The area and stiffness of the rods could be tuned to achieved a different overall stiffness within the connection region. This technique was applied for a few test cases, but it was found to cause numerical errors when performing some nonlinear static deflection tests and modal analysis tests. For this reason, the rigid RBE2 elements were used for the majority of the work presented in this thesis. Use of actual connection regions through the Nastran BCBODY and BSURF 
commands may be worth re-exploring for a more realistic representation of the actual vehicle.

The connections representing the servos of the control surfaces were modeled in Nastran by carefully examining the defined connections in the original ANSYS model. Corresponding rigid and flexible bar connections were created to match the ANSYS model. The servo connecting nodes were allowed to rotate around each other in order to simulate actual control surface deflection.

\section{A.5 Model Verification}

Once the complete model had been converted to Nastran, a verification step was performed to gauge the accuracy of the conversion process. A modal analysis was performed on both the ANSYS and Nastran models, and the natural frequencies of each model were compared. Once the error in the frequencies was acceptable, the model was deemed adequately converted. Table A.1 compares the first six elastic frequencies after the conversion process.

Table A.1: Results of the finite element model conversion.

\begin{tabular}{|c|c|c|c|}
\hline Property & ANSYS model value & Nastran model value & \% difference \\
\hline Elastic mode $1 \omega(\mathrm{Hz})$ & 9.746 & 9.278 & $-4.802 \%$ \\
\hline Elastic mode $2 \omega(\mathrm{Hz})$ & 14.763 & 12.983 & $-12.057 \%$ \\
\hline Elastic mode $3 \omega(\mathrm{Hz})$ & 19.303 & 18.598 & $-3.791 \%$ \\
\hline Elastic mode $4 \omega(\mathrm{Hz})$ & 23.335 & 21.795 & $-6.600 \%$ \\
\hline Elastic mode $5 \omega(\mathrm{Hz})$ & 28.094 & 27.416 & $-2.413 \%$ \\
\hline Elastic mode $6 \omega(\mathrm{Hz})$ & 35.978 & 34.474 & $-4.180 \%$ \\
\hline
\end{tabular}




\section{Appendix B}

\section{High Fidelity Flight Test Modeling}

Parallel to the aeroelastic scaling work presented in this thesis, the converted Nastran finite element model of the flight test RPV was used to conduct high fidelity modeling tests. Some of these tests and validations were concurrently run in ANSYS to provide assurance that the models were producing acceptable results. This appendix documents the most noteworthy high fidelity tests that were performed using the converted Nastran model in order to aid the RPV flight test effort. The main goals of the high fidelity modeling effort were as follows:

1. Attempt to identify the nonlinear response desired in the flight test

2. Determine which aircraft components or regions require redesign

3. Propose and test new designs of these components before manufacturing 


\section{B.1 Early Design Exploration}

To act as a starting point for the high fidelity tests, a low fidelity representative beam model of the RPV was created by Anthony Ricciardi. A parametric study on this model that varied beam and joint stiffnesses concluded that a rotational "leaf" spring at the fuselage/forward wing joint and a soft aft wing would produce the greatest nonlinear response. These design changes were implemented into the high fidelity model for initial testing.

To quickly model a soft aft wing, materials in the aft wing structure (not including the control surfaces) were singled out. The elastic modulus of these materials was then multiplied by a scaling factor in order to reduce the overall stiffness of the entire aft wing. As a base case, the aft wing was taken to $5 \%$ of the original stiffness.

The rotational "leaf" spring was modeled by defining coincident nodes at the fuselage/forward wing joint. Connection nodes on the fuselage were tied to one coincident node while connection nodes of the wing were tied to the other. The coincident nodes were connected using a torsional spring element constrained to rotate about defined local coordinate systems. These coordinate systems provided the correct bending direction of the forward wing. The element spring stiffness $k$ could be tuned to change the stiffness of the joint connection. Figure B.1 shows the rotational "leaf" spring within the high fidelity model. 


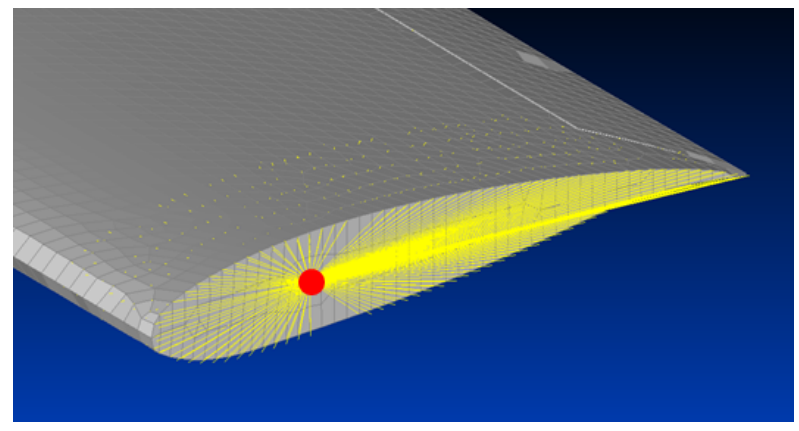

(a) Wing connections to joint node.

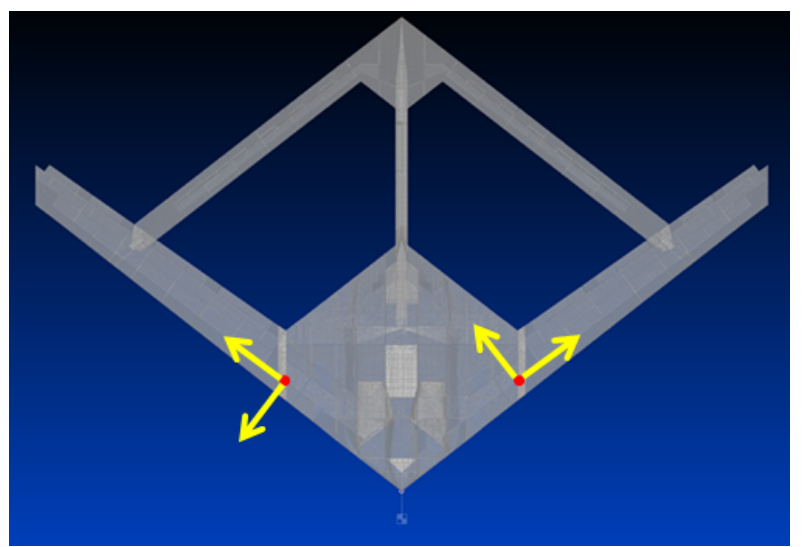

(b) Local coordinate systems.

Figure B.1: Rotational "leaf" spring joint.

A static $2.5 \mathrm{~g}$ trim load case calculated from the low fidelity beam model was used to test the design. The test was conducted using $5 \%$ aft wing stiffness and $k=1 \times 10^{7} \mathrm{~N} \cdot \mathrm{mm}$. Figure B.2a shows the linear static results, and figure B.2b shows the nonlinear static results.

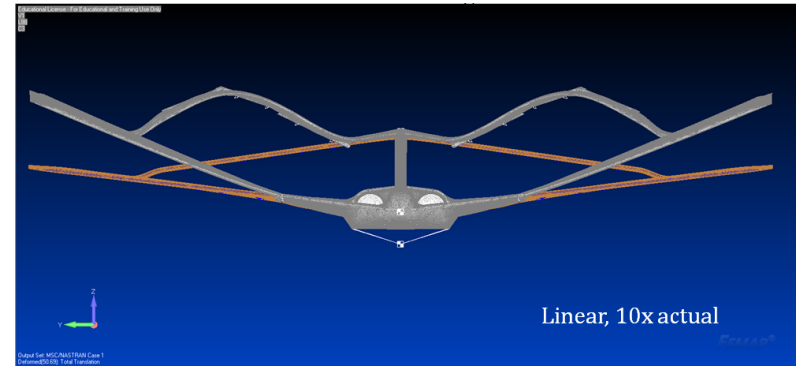

(a) Linear static deflection.

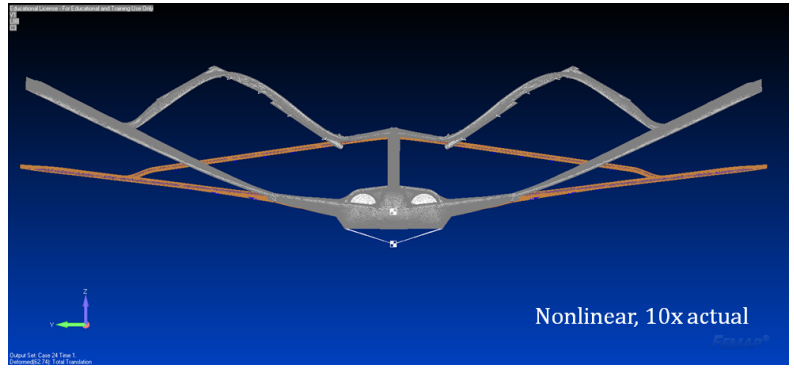

(b) Nonlinear static deflection.

Figure B.2: 2.5g trim load static deflection results with soft aft wing and spring joint.

The relative vertical deflection of the mid point of the aft wing measured from a straight line between the wing end points was calculated in each case. In the linear case, the relative deflection was $28.5 \mathrm{~mm}$. In the nonlinear case, the relative deflection was $38.3 \mathrm{~mm}$, an increase of about 34\%. These results indicated that softening the aft wing would indeed 
produce a nonlinear response that was appreciably different than the linear response. The deflection shapes themselves were never meant to be representative of the nonlinear response expected in flight, however. This quick, qualitative test was only meant to show that a nonlinear response could be achieved through the softening of the aft wing and inclusion of a "leaf" spring joint.

\section{B.2 Aft Wing Ladder Structure}

Subsequent parametric tests and manufacturing concerns suggested that the "leaf" spring design may not have been as beneficial as originally thought. Thus, the next set of design efforts was concentrated on the design of a softer aft wing. Ricciardi designed a ladder beam structure to serve as the load bearing member of the aft wing. Forward and aft spars were connected with evenly spaced rungs, and an aluminum alloy was chosen as the material. Cross sectional properties of the spars and rungs could be designed. Concentrated ballast masses were placed along the length of the ladder.

After successful initial tests on the low fidelity beam model, the ladder structure was implemented into the high fidelity model. The original aft wing was removed and replaced with the beam ladder structure. The aerodynamic shell that would surround the ladder was assumed to carry no load and was neglected from the model. Figure B.3 shows the integrated ladder structure into the high fidelity model. 


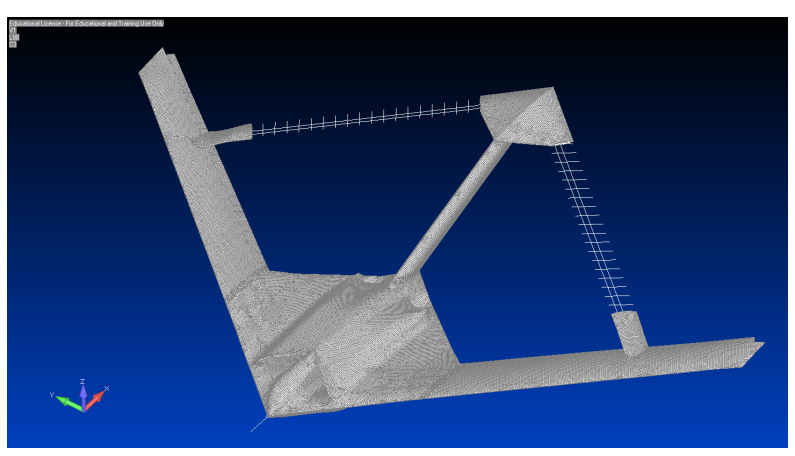

Figure B.3: Aft wing ladder beam structure integrated into high fidelity model.

With the integration of the designable ladder structure, various cross sectional properties could be tested on the high fidelity model. Research continues in an attempt to find the design that produces a measurable nonlinear response in flight.

\section{B.3 Model Tuning and Forward Wing Testing}

If the aft wing was made extremely flexible, a greater portion of the load during flight would be carried in the forward wing. Concerns arose as to whether or not the existing forward wing could carry the added load without failing. An extensive experiment was conducted on the ANSYS model and flight test RPV to determine the feasibility of keeping the current forward wing. ${ }^{20}$ Portions of the study were mirrored on the Nastran model as a verification.

The Nastran model was first updated and tuned to better match the latest experimental results. An optimization was run in order to tune the materials within the model to match a static tip deflection. For these tests, the aft wing was removed completely, and a point load was applied to the aft wing connection joint. The results of the initial tuning are shown 
below. Figure B.4 compares the wing tip deflections of the tuned ANSYS and Nastran models and the RPV. Figure B.5 compares the root deflections. Up to a load of approximately 10 $\mathrm{kg}$, all of the models agree reasonably well. See Reference 20 for more extensive details on the experimental set-up and execution.

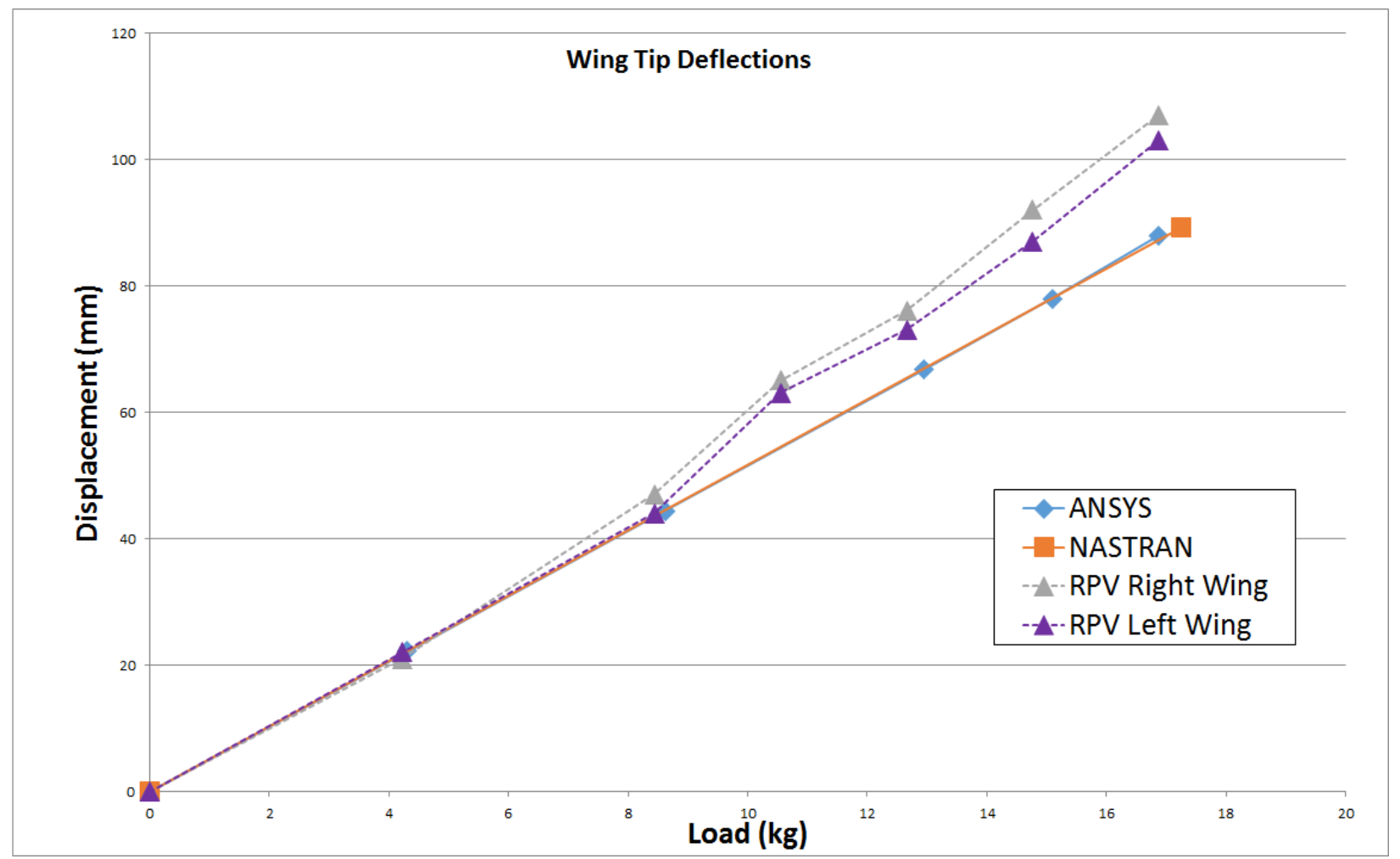

Figure B.4: Wing tip deflections after model tuning. 


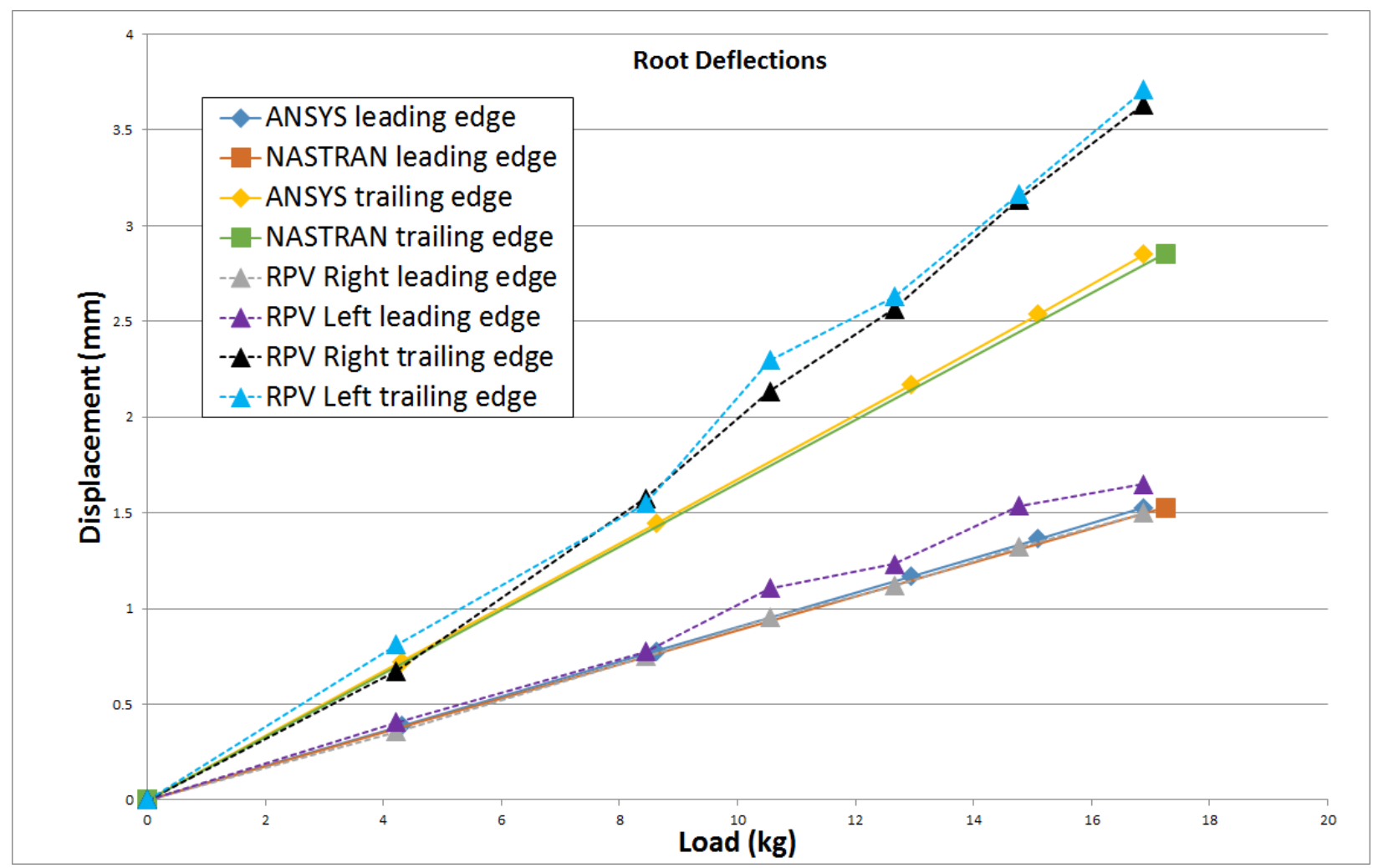

Figure B.5: Root deflections after model tuning.

In order to better capture the strain profile of the forward wing, fillets were added to the top and bottom of the shear web spars. These fillets were modeled by adding a layer onto the bottom of the ply elements along the side of each shear web. The thickness of the fillets could be changed to better tune the model to the experimental results and were given an initial thickness of $8 \mathrm{~mm}$. Figure B.6 shows the locations of the added fillets in one of the forward wings. 


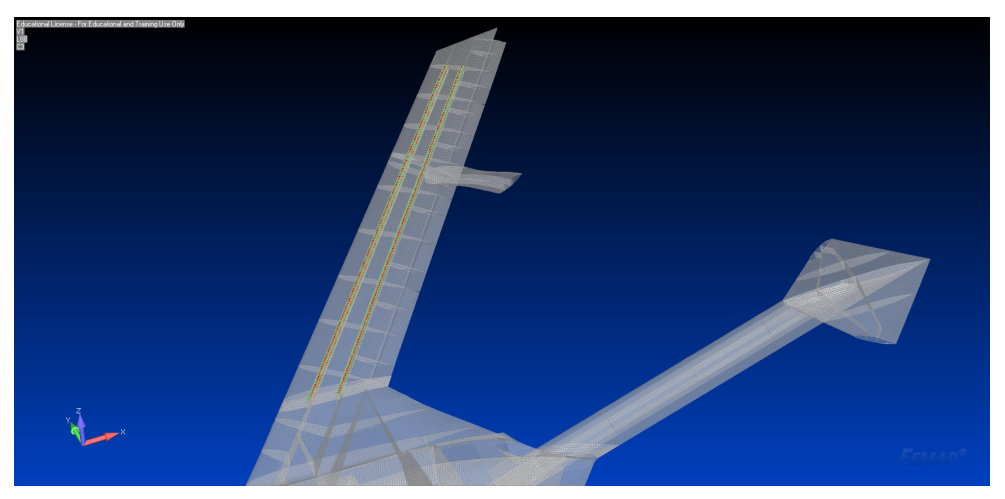

Figure B.6: Fillet locations in forward wing.

Strain results were compared between the models. Major and minor principal strains were measured at the root of the forward wing at the top of the leading edge shear web, trailing edge shear web, and middle depression. Figure B.7 shows the major principal strain profile of the top ply layer at a load of $13.8 \mathrm{~kg}$ and the locations of the strain measurements. Figures B.8, B.9, and B.10 compare the measured strains from the leading edge, trailing edge, and middle region, respectively.

Up to a load of approximately $10 \mathrm{~kg}$, the models agree very well along the leading and trailing edges. Some significant difference is seen in the middle strain region, however. Thus, the model was considered reasonably tuned within the linear experimental region up to a load of $10 \mathrm{~kg}$. Extensive panel buckling at higher load steps was not accurately captured.

The tuned Nastran model agrees reasonably well with the ANSYS model used in the experimental study of Reference 20. As concluded in this work, the existing forward wing is not suited to carry a flight load without the presence of a load bearing aft wing. Thus, research efforts are now concentrating on redesigning both the forward and aft wings in order 
to produce a measurable nonlinear response in flight. These new designs can be integrated into the high fidelity Nastran model for testing.

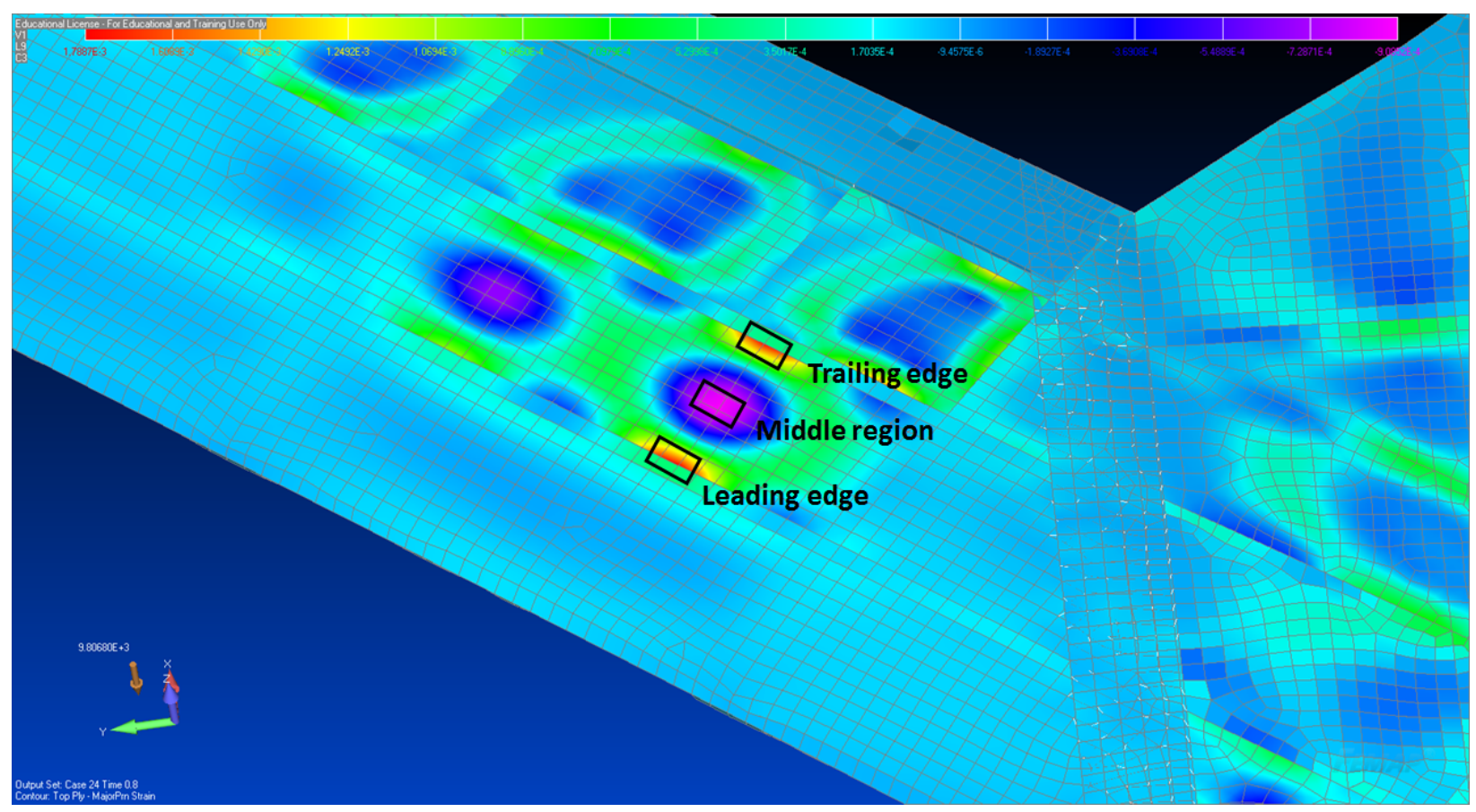

Figure B.7: Major principal strain of top ply layer at $13.8 \mathrm{~kg}$ and measurement locations. 


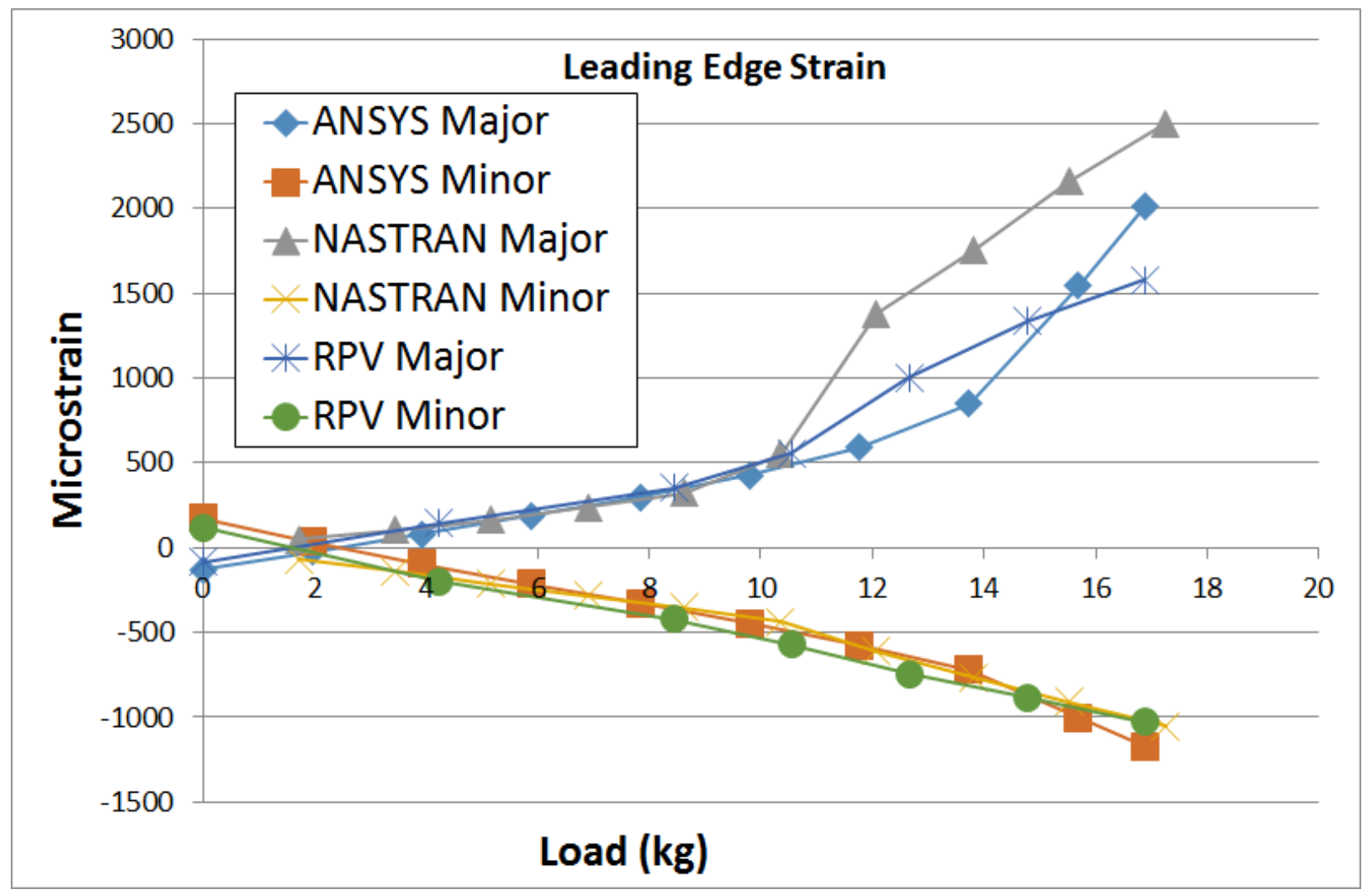

Figure B.8: Leading edge strain measurements after model tuning. 


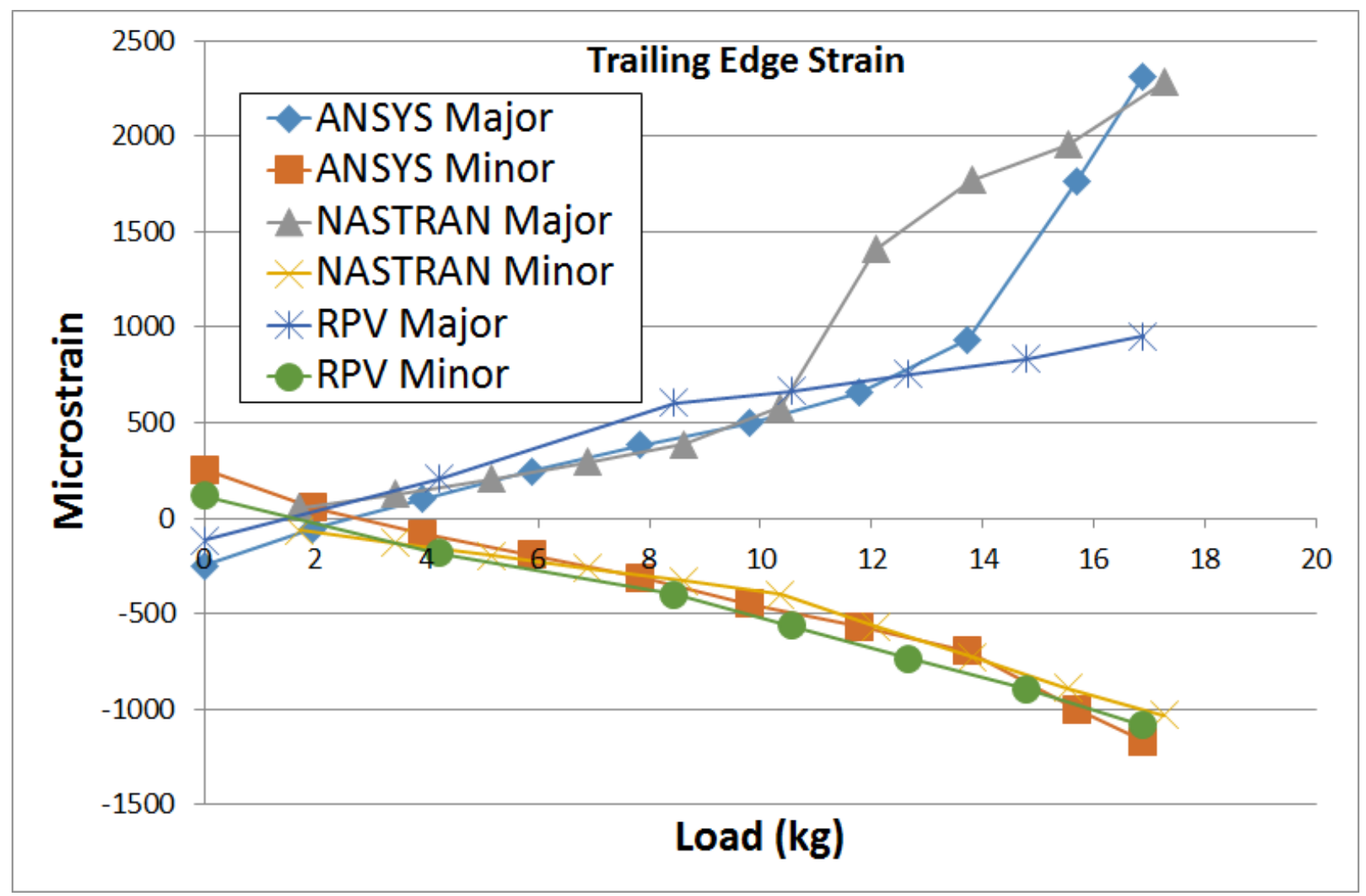

Figure B.9: Trailing edge strain measurements after model tuning. 


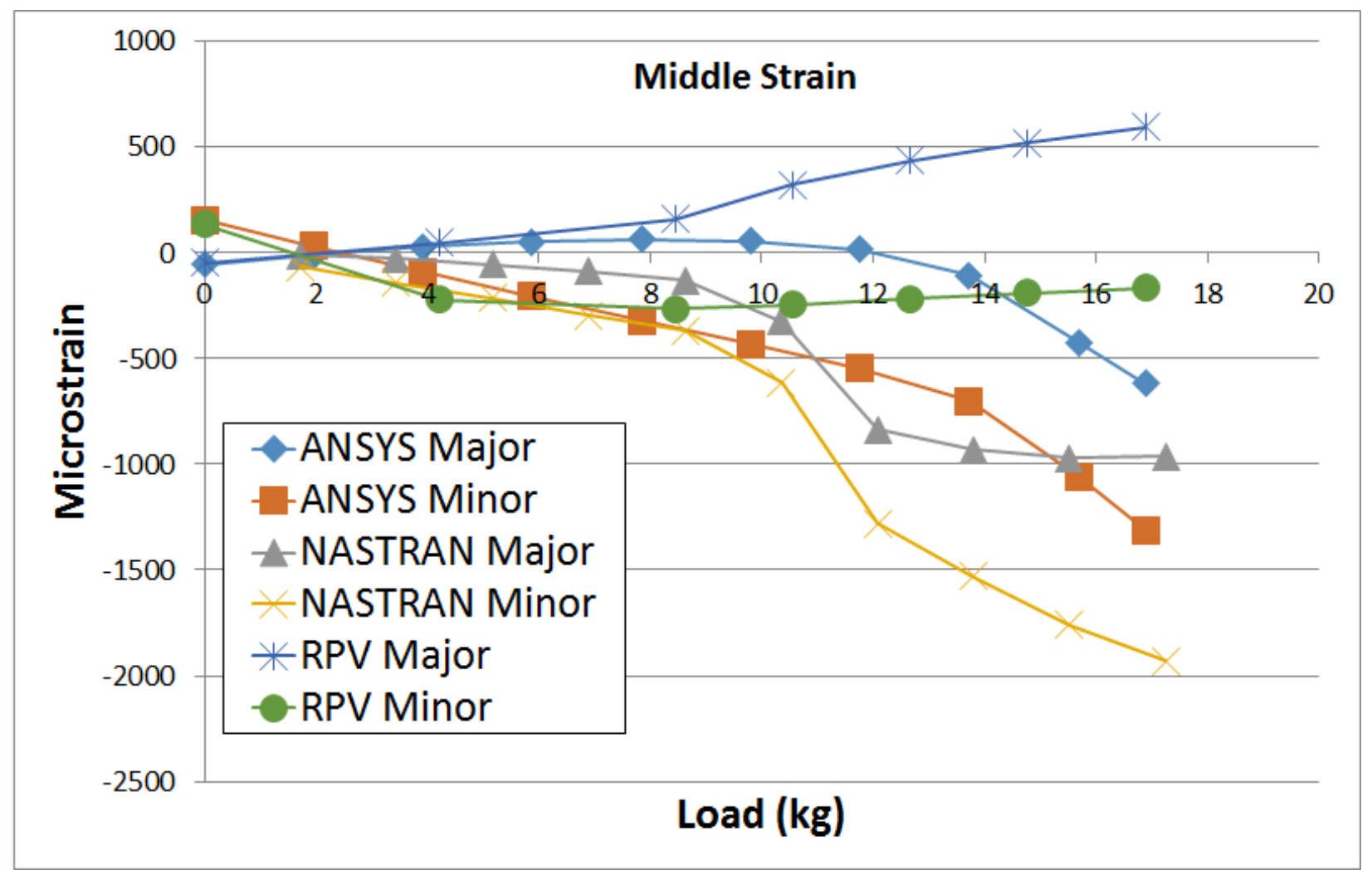

Figure B.10: Middle region strain measurements after model tuning. 